\title{
Untangling the Reciprocal Relation of the Behavioral and Physiological Immune Systems
}

\author{
Baris Sevi \\ West Virginia University, bs0059@mix.wvu.edu
}

Follow this and additional works at: https://researchrepository.wvu.edu/etd

Part of the Biological Psychology Commons

\section{Recommended Citation}

Sevi, Baris, "Untangling the Reciprocal Relation of the Behavioral and Physiological Immune Systems" (2019). Graduate Theses, Dissertations, and Problem Reports. 4101.

https://researchrepository.wvu.edu/etd/4101

This Thesis is protected by copyright and/or related rights. It has been brought to you by the The Research Repository @ WVU with permission from the rights-holder(s). You are free to use this Thesis in any way that is permitted by the copyright and related rights legislation that applies to your use. For other uses you must obtain permission from the rights-holder(s) directly, unless additional rights are indicated by a Creative Commons license in the record and/ or on the work itself. This Thesis has been accepted for inclusion in WVU Graduate Theses, Dissertations, and Problem Reports collection by an authorized administrator of The Research Repository @ WVU. For more information, please contact researchrepository@mail.wvu.edu. 
Untangling the Reciprocal Relation of the

Behavioral and Physiological Immune Systems

\author{
Barış Sevi
}

\author{
Thesis submitted \\ to the Eberly College of Arts and Sciences \\ At West Virginia University

\begin{abstract}
in partial fulfillment of the requirements for the degree of
Master of Science in Psychology
\end{abstract}
Natalie J. Shook, PhD., Chair
Steven G. Kinsey, PhD.
Melissa Blank, PhD.

\title{
Morgantown, WV
}

2019

Keywords: Behavioral immune system, physiological immune system, recent illness, IL-6 Copyright 2019 Barış Sevi 


\begin{abstract}
Untangling the Reciprocal Relation of the Behavioral and Physiological Immune Systems
\end{abstract}

\title{
Barış Sevi
}

In the past decade, there has been an exponential increase in the study of the Behavioral Immune System (BIS), a suite of psychological processes that serves an infectious disease avoidance function. The BIS is proposed to be inherently intertwined with the physiological immune system. However, very few studies have actually demonstrated this fundamental, theoretical assumption. The purpose of this thesis was to test the possible relation between the physiological and behavioral immune systems in two studies. In Study $1(N=388)$, the relation between implied activation of the physiological immune system and BIS sensitivity was examined. Participants who reported more recent illness or having experienced more non-infectious illness in their past perceived themselves as more vulnerable to disease but did not report greater levels of disgust sensitivity. In Study 2, the effect of BIS activation on physiological immune response was tested. Participants $(N=45)$ were randomly assigned to either a disease threat or a non-disease threat condition. Before and after exposure to the respective priming materials, participants provided saliva samples that were assayed for proinflammatory cytokine interleukin-6 (IL-6). Condition did not significantly affect IL-6 levels. However, these results should be interpreted with caution, as IL-6 levels in the majority of the samples were undetectable, which resulted in an underpowered sample $(n=$ 16). Overall, these findings do not support the assumption that the behavioral immune system and the physiological immune system are associated 


\section{Table of Contents}

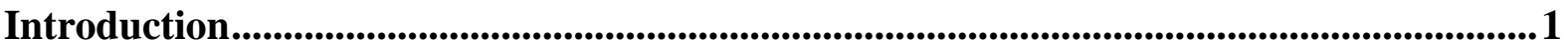

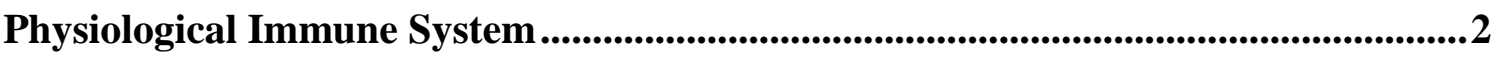

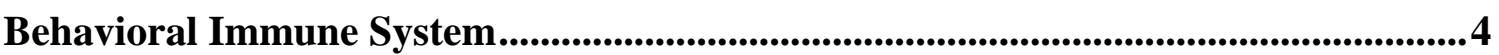

Connection Between the Behavioral and the Physiological Immune Systems.............7

Current Reserach ................................................................................................................

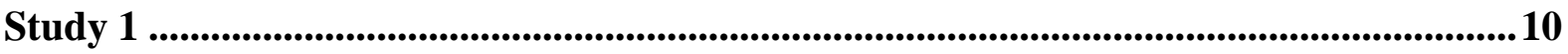

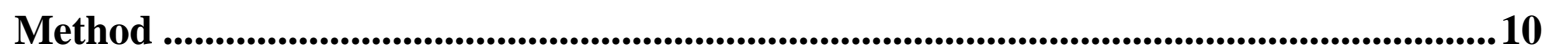

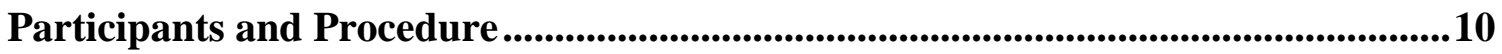

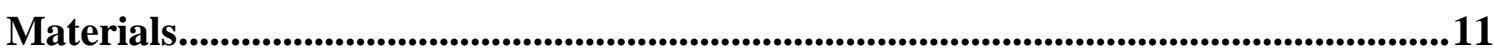

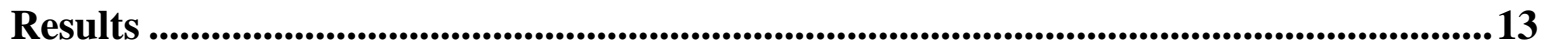

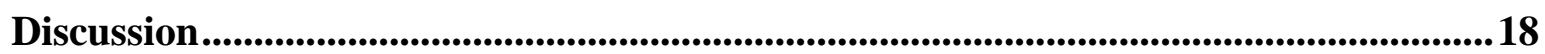

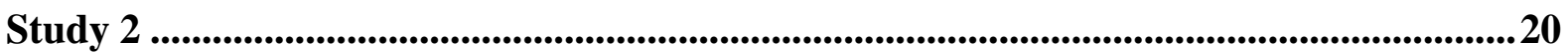

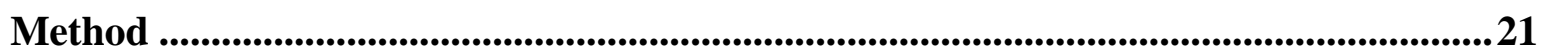

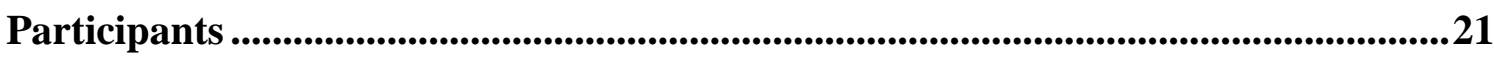

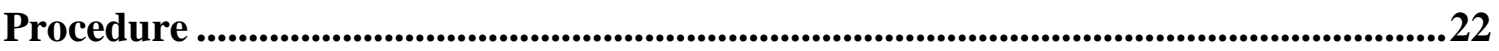

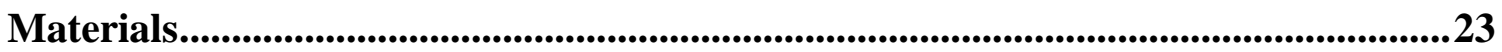

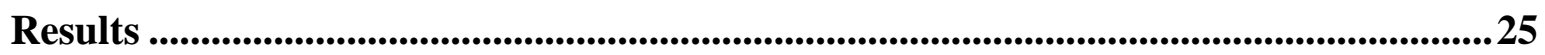

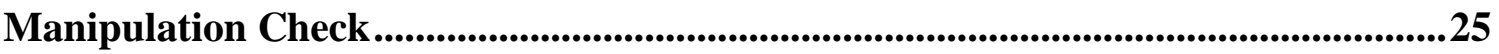

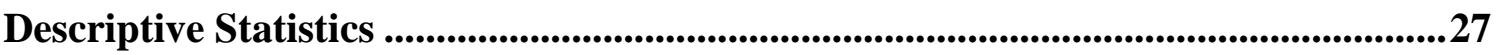

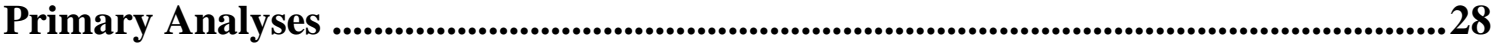

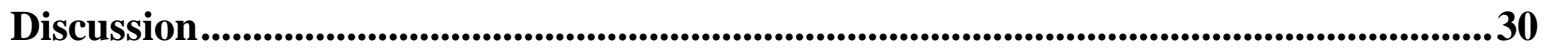

General Discussion............................................................................................................................332

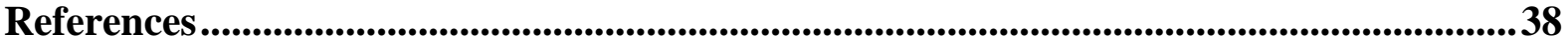

Appendix A ...........................................................................................................................................446

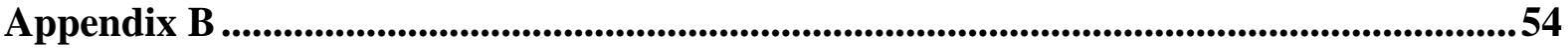

Appendix C .............................................................................................................................................55 


\section{Introduction}

People face many different threats (e.g., predators, climate changes) to their survival and successful reproduction. In order to counter these threats, physiological and psychological mechanisms have been shaped by evolutionary processes to produce the "best" mechanisms to help individuals avoid death, reproduce, and pass on their genes (Neuberg, Kenrick, \& Schaller, 2010). Infectious diseases have been one of the most significant threats to the health and fitness of humans (Schaller \& Murray, 2008). As a recent example, "The plague" during the 14th century caused the death of 30-60\% of Europe's population (Suzanne, 2003). To increase the adaptive fitness of the human body, different systems that are comprised of the collaboration of different mechanisms have evolved. The most known of these systems, the physiological immune system evolved to combat parasites, infection, and disease. Although effective, the physiological immune system is a metabolically costly safeguard against infectious diseases (Schaller \& Park, 2011). To minimize these metabolic costs, a set of psychological mechanisms also evolved to prevent contact with pathogens and disease transmission. Schaller and Duncan (2007) coined the phrase "Behavioral Immune System," or BIS, to describe these psychological processes that work together as the first defense barrier against infectious diseases. To be consistent with the existing literature and avoid confusion, the same terminology is used in this document to distinguish the psychological and physiological components of the immune system (i.e., behavioral immune system and physiological immune system, respectively).

Theoretically, the physiological immune system and behavioral immune system are complementary and inherently linked. However, very little research has examined if these systems are related, and the existing evidence has utilized variable methods and measures. The purpose of this project was to test the assumed connection between these psychological and physiological processes. Specifically, two studies were conducted to determine if the 
physiological immune system and the biological immune system are related. In the first study, the relation between implied activation of the physiological immune system (i.e., illness history) and BIS sensitivity was examined. In the second study, the effect of BIS activation (i.e., disease-threat prime) on physiological immune system response was tested. It was hypothesized that activation of one system would be associated with activation of the other system.

\section{Physiological Immune System}

Pathogens are biological agents (e.g., viruses, bacteria, fungi, protozoans, worms) that can produce disease. There are a number of ways that pathogens can be transmitted. However, a primary source of pathogen transmission is through either direct (e.g., physical contact, sneezes, coughs) or indirect (e.g., touching a contaminated surface) contact with other people (Loh et al., 2015). If pathogens cannot be repelled before entrance into the body, they try to establish a site for infection. That is, they will invade the host's body, multiply, release toxins, and destroy host cells, which results in infectious disease (Janeway et al., 2001). Thus, infectious disease is any disorder that disables normal functioning and is caused by pathogens.

The physiological immune system is a complex group of defense responses that helps to protect against pathogens and the emergence of infectious diseases. The physiological immune system consists of two cooperative defense systems: specific (acquired immunity) and nonspecific (innate immunity; Diercks \& Kluin, 2016). The specific immune system reacts to particular types of pathogens and is acquired through exposure to the infectious agent. The nonspecific system defends against all pathogens equally and functions as the first response against infectious diseases (Delves \& Roitt, 2000).

The specific system can distinguish between different infiltrators, developing a unique response that adapts to specific pathogens, called antigens (Abbas, Lichtman, \& Pillai, 2014). 
Importantly, other than exceptions like autoimmune diseases or eliminating damaged cells, this system tolerates the self and does not react in a harmful way towards the self. Lymphocytes are types of white blood cells that aid in eliminating pathogens inside the body and are an essential part of the specific system. The specific system can be divided into two forms of immunity (i.e., humoral and cell-mediated immunity) according to the types of lymphocytes involved in responding to infection. Humoral immunity is operated by B lymphocytes, which produce proteins called antibodies that tag antigens so that they may be attacked by the other parts of the immune system. The function of humoral immunity is to fight against pathogens and toxins outside of the host's cells. Cell-mediated immunity is operated by T lymphocytes, which recognize and kill pathogen-infected cells. The function of the cell-mediated immunity is to destroy the pathogens inside of the infected cells.

Despite the effectiveness of a specific system, there is a diverse variety of pathogens that are encountered on a regular basis. A nonspecific system that defends against all types of disease threat equally is also beneficial for an organism. The nonspecific system has three principal components: Physical barriers (e.g., epithelia), cellular bodies (e.g., phagocytes and natural killer cells), and fluid-phase molecules (e.g., acute phase proteins). The first response that this system creates to fight pathogens is inflammation (Kawai \& Akira, 2006). Cytokines synthesized to aid in defending the body against diseases when an injured cell or a foreign substance is detected produce inflammation (Heinrich et al., 2003). Cytokines are small proteins essential in cell signaling and communication between cells. They mediate and regulate the nonspecific system as well as the specific immune system, and stimulate hematopoiesis (Abbas, Lichtman, \& Pober, 2000). The types of cytokines in the nonspecific immune system vary according to their function and structure. For example, proinflammatory cytokines are responsible for the emergence of inflammation. Inflammation increases blood flow, including white blood cells, to the damaged or infected tissue and can be observed by 
redness, swelling, heat, and pain in the area (Maier \& Watkins, 1998). This helps to isolate the infection from further contact with body tissues, allowing "professional phagocytes" such as macrophages and monocytes to destroy the infiltrating pathogens by ingesting them through the process of phagocytosis (Abbas et al., 2014; Gordon, 2016; Mandell, 1995).

Generally, the physiological immune system is effective, but there are limitations. First, it is a reactive system that responds only after contact with the infectious agent (Miller \& Maner, 2011). Second, operations of the physiological immune system that fight infections, like inflammation and fever, have high metabolic costs (Baracos, Whitmore, \& Gale, 1987). Third, in cases of malfunction the physiological immune system can develop an autoimmune disorder and attack its own healthy tissues (Vyse \& Todd, 1996). Further, the reactions of the physiological immune system can have effects on social behavior. For example, to mitigate further damage and conserve energy social withdrawal increases and other behaviors such as exploration and foraging decrease (Ackerman, Hill, \& Murray, 2018). These behavioral changes are maladaptive as they may leave the individual vulnerable to other survival threats (e.g., predators). To compensate for these weaknesses, the behavioral immune system (BIS) evolved as a complementary "first line of defense" against pathogen and parasitic infection (Schaller, 2006).

\section{Behavioral Immune System}

The BIS is a suite of psychological processes that evolved to serve a diseaseavoidance function. Specifically, the BIS consists of affective, cognitive, and behavioral processes that detect and respond to potential sources of contamination, generally by preemptively distancing individuals from the perceived source of infection (Schaller \& Duncan, 2007). The BIS is activated by sensory stimuli that indicate a potential disease threat (e.g., vomit, feces). This sensory input evokes emotional and cognitive processes that lead to prophylactic behaviors (e.g., avoidance). Perceiving disease cues and eliminating possible 
contamination are essential parts of the BIS. Therefore, people can quickly detect morphological cues of infection (e.g., rashes), and display behavioral reactions to those cues (Ackerman et al., 2009). Through the early detection and avoidance of potential contaminants, the likelihood of exposure to pathogens and infection is reduced, and the physiological immune system is not fully engaged, thus preserving physiological resources.

Disgust is a primary affective component of the BIS that is thought to defend against infectious diseases (Faulkner, Schaller, Park, \& Duncan, 2004). This emotion is aroused by visual, olfactory, tactile, gustatory, and auditory cues that signal the potential presence of pathogens (e.g., look of an open wound, taste of sour milk). Arousal of this emotion urges the individual to be aware and keep a distance from the possible source of pathogens, thus reducing possible contamination (Oosterhoff, Shook, \& Ford, 2018). Disgust is a universal emotion that most individuals experience (Tybur et al. 2016); however, there is individual variability in sensitivity to disgust (Tybur et al., 2009). Greater disgust sensitivity is characterized by attitudes and behavior that motivate the individual to avoid possible sources of disease. For example, people with higher disgust sensitivity show higher levels of food neophobia (Al-Shawaf, Lewis, Alley, \& Buss, 2015), which encourages avoidance of possible contaminants or sickness from a new food. Like food, people are also possible carriers of pathogens. Accordingly, people with higher disgust exhibit less trust towards others (Aarøe, Osmundsen, \& Petersen, 2016).

Another indicator of the BIS is perceived vulnerability to disease (PVD; Duncan, Schaller, \& Park, 2009). Perceived vulnerability to disease is a chronic individual difference variable consisting of two dimensions: germ aversion and perceived infectability. Germ aversion refers to the emotional discomfort one experiences in a situation with a high risk of pathogen transmission. Perceived infectability refers to an individual's belief in his or her own susceptibility to infectious diseases. Having high levels of PVD serves to decrease 
possible contamination risks. For example, PVD enhances the effects of perceived disease cues. When primed with images of sick people, individuals higher in PVD reacted faster to images of faces by pushing away from the images, a behavior that serves to avoid potential infection sources (Mortensen, Becker, Ackerman, Neuberg, \& Kenrick, 2010). Further, PVD has been related to judgment of attractiveness; when asked to rate the attractiveness of faces in a movie clip, participants with greater PVD showed a preference for healthy looking faces (Welling, Conway, DeBruine, \& Jones, 2007).

The main evolved function of the BIS is to protect the body from pathogens, but as with many evolved mechanisms, there are byproducts to the adaptive function. For the BIS, these byproducts have fallen in the social realm of people, such as outgroup behavior and personality (Leeuwen \& Petersen, 2017). Prejudice against individuals who are perceived to be potential carriers of pathogens or parasites has an adaptive utility of avoiding possible harmful pathogens and parasites that can be transmitted through contact (Kurzban \& Leary, 2001; Park, Faulkner, \& Schaller, 2003). Faulkner et al. (2004) suggested that in ancestral environments, where there were many hazardous pathogens for human health, an adaptive tendency would have been to avoid individuals who were possible carriers of contagious diseases. Therefore, xenophobic attitudes are observed when there is a disease threat. In the same vein, it has been shown that extraverted people, who are at high risk of pathogen transmission, tend to be less aversive towards germs than introverted people, and are fewer in places with high prevalence of infectious diseases (Hamrick, Cohen, \& Rodriguez, 2002; Duncan, Schaller, \& Park, 2009; Schaller \& Murray, 2008).

In the past decade, there has been an exponential increase in BIS research. When the term "Behavioral Immune System" is searched in PsycINFO 80\% of the published papers are from the last five years (i.e., 2013-2018). However, most of this work has investigated the social byproducts of this system, whereas the study of the main function of the BIS has fallen 
behind, especially its possible relation with the physiological immune system (see Ackerman et al., 2018, for a review). Theoretically, the behavioral immune system and physiological immune system are complementary and inherently linked. Yet, very little research has examined if these systems are related or not. The existing evidence has used variable methods and outcome measures, which does not provide a robust finding. A better understanding of the BIS as an evolved mechanism is essential for evaluating the validity and reliability of the existing research. Thus, there is an important gap in the literature.

\section{Connection Between the Behavioral and the Physiological Immune Systems}

The connection between the physiological immune system and psychological factors is not new. Extensive work in the field of psychoneuroimmunology has documented the relation between physiological immune markers and psychological processes (e.g., Clark \& Fessler, 2014; Maier \& Watkins, 1998; Miller \& Cohen, 2001). For example, greater loneliness and small social networks are associated with poorer antibody response (Pressman et al., 2005), and relaxation training increased specific immune responses in an elderly sample (Kiecolt-Glaser et al., 1985). To date, however, very few studies have specifically examined a relation between the BIS and physiological immune function.

There are two general ways that have been used to study a relation between the behavioral and physiological immune systems. The first way has been to assess the relation between recent physiological immune system activity and indicators of the behavioral immune system. Miller and Maner (2011) demonstrated that individuals who had been ill more recently (within the past week) exhibited an attention bias toward disfigured faces versus normal faces and were faster to avoid (vs. approach) images of disfigured (vs. normal) faces. Participants who had not been ill recently did not show any attention or approach/avoidance biases. In another study, men with higher testosterone and lower cortisol, qualities that are shown to be associated with stronger physiological immune system 
responses, presented less aversion towards faces that depicted color cues associated with high vulnerability to infectious disease (Kandrik et al., 2017). Arguably differences in testosterone and cortisol are related to a less reactive behavioral immune system response through their association with the physiological immune system. As another example, women in the first trimester of pregnancy, when maternal immune function is most suppressed, tended to report more disgust sensitivity than those in the second or third trimester (Fessler, Eng, \& Navarrete, 2005). Thus, activation or weakening of the physiological immune system seems to be related to increased sensitivity of the behavioral immune system.

The second way to study the relation between the two systems has been to test the effect of BIS activation on the physiological immune system. The primary immune markers of interest have been cytokines, because they are part of the non-specific immune system and are secreted by tissue cells when there are possible pathogen threats (Abbas et al., 2014). In particular, previous studies examining the influences of BIS on the physiological immune system have used the proinflammatory cytokines interleukin-6 (IL-6) and tumor necrosis factor alpha (TNF-a) to assess the activation of the physiological immune system.

Schaller, Miller, Gervais, Yager, and Chen (2010) were the first to investigate whether activating the BIS would lead to changes in physiological immune response. In their study, participants were randomly assigned to either a disease threat (e.g., skin lesions, sneezing) or non-disease threat (e.g., person pointing a gun) priming condition. Blood samples were collected before and after the priming procedure to assess whether levels of IL6 changed in response to exposure to a visual disease threat. Participants in the disease threat condition exhibited a significant increase in IL-6, whereas IL-6 did not significantly increase in the non-disease threat condition. This finding was the first to demonstrate the complementary nature of the BIS and the physiological immune system.

A few studies have tested the relation between disgust (an affective component of the 
BIS) and activation of the physiological immune system. Stevenson and colleagues (2011) demonstrated that inducing disgust in males with visual stimuli resulted in an increased oral inflammatory response (i.e., increased levels of TNF-a and albumin levels) as compared to control groups. Similarly, inducing disgust in males led to an increase in core body temperature compared to a control group (Stevenson et al., 2012). However, Stevenson and colleagues (2015) did not find a significant simple effect of priming males with disgust or disease-threat images on physiological immune markers (i.e., salivary secretory immunoglobulin A (sIgA) and TNF-a). Rather, disgust sensitivity moderated the relation, such that participants who were higher in disgust propensity showed greater TNF-a and sIgA responses to disgusting or disease-related images compared to negative images.

Overall, these studies provide evidence suggesting a link between the BIS and the physiological immune system. However, there are limitations to these studies. First, these studies have generally examined different immune markers and/or different means of activating the BIS. Thus, the literature lacks direct replication of key findings to demonstrate the fundamental assumptions of the BIS theory. Second, the studies that examined the same markers have also shown inconsistent results, which raises concern for the reliability of the relation between the two systems. Consistency in results is essential to create an empirical base for the BIS theory and move the field forward.

\section{Current Reserach}

A fundamental assumption of the BIS theory is that the physiological immune system and the BIS are interconnected. However, very few studies have actually tested this assumption and there are limitations to this existing work. The strength of these findings has great importance for the future of BIS research. Thus, the goal of the proposed research was to investigate the potential connection between the behavioral and physiological immune systems, while addressing some of the limitations of previous work. Specifically, two studies 
were conducted. In the first study, the relation between implied physiological immune system activity and BIS sensitivity was assessed. In the second study, the effect of BIS activation on physiological immune system function was tested. For the first study, it was expected that greater implied activation of the physiological immune system would be related to greater activation of the BIS. In the second study, it was hypothesized that when the BIS is activated the physiological immune system would also be activated.

\section{Study 1}

The goal of the first study was to examine whether greater implied activation of the physiological immune system is related to a greater BIS sensitivity. The behavioral immune system's first objective is to defend against contact with pathogens and avoid infectious diseases. If a person has experienced more infectious disease, it is logical to expect that their BIS should be more sensitive. In this study, illness history and recency of illness were used as indices of physiological immune system activation, and individual differences in disgust sensitivity and perceived vulnerability to disease were assessed as BIS indicators.

\section{Method}

\section{Participants and Procedure}

As there was no prior research similar to this study, a small effect $(f)$ of .1 was expected as a conservative estimate, which required a minimum of 143 participants to attain $80 \%$ power with an alpha of .05 for a multiple regression analysis with six variables. A total of 403 WVU students (321 women; age range 18 - 35 years, $M_{\text {age }}=19.71 S D_{\text {age }}=1.84$; 85.4\% Caucasian; $86.6 \%$ Heterosexual; $M d n_{\text {income }}=\$ 80,000$ - $\left.\$ 99,999\right)$ from the Department of Psychology's subject pool participated in the study. All students who were 18 years old or older were eligible to participate. Data from some participants were excluded from the analyses for various reasons: not fully completing the measures $(n=5)$, completing the study 
twice $(n=4)$, and outlier scores $(n=2)$. Excluded participants were compared to retained participants on demographics and primary variables. For participants who completed the study twice, their first responses were used. Overall, excluded participants did not significantly differ from retained participants on most demographic variables or primary variables $(p s>.05)$, except for illness recency and non-infectious disease history. Excluded participants reported more recent illness $\left(t(390)=-2.81, p=.01 ; M_{\text {retained }}=2.90, M_{\text {excluded }}=\right.$ 5.12) and less non-infectious disease in their history $\left(t(378)=2.47, p=.01 ; M_{\text {retained }}=2.63\right.$, $\left.M_{\text {excluded }}=.50\right)$ compared to the retained sample. The final sample consisted of 388 participants (306 women; age range 18 - 35 years, $M_{\text {age }}=19.74, S D_{\text {age }}=1.86$ ).

The study was conducted through the online survey system Qualtrics. Participants were redirected to the study page through a link on SONA. After agreeing to an online consent form, participants completed the following questionnaires in a random order (see Appendix A for complete measures). After the study was finished, participants were compensated with course credit.

\section{Materials}

Recent Illness. Recency of illness was assessed with five items (Miller \& Maner, 2011). Participants indicated the last time they had a cold by choosing from seven response options: "today," "a couple days ago," "a week ago," "a couple weeks ago," "a month ago," "a few months ago," and "a year or more ago.” Based on Miller and Maner (2011), participants were sorted into two groups according to their answers: recently ill participants (responded with "today," "a couple days ago," or "a week ago,") or not recently ill participants (responded with "a month ago," "a few months ago," or "a year or more ago").

Participants also indicated their agreement on a 7-point scale from 1 ("strongly disagree") to 7 ("strongly agree") with four statements: "Over the past couple days, I have not been feeling well"; "Lately, I have been feeling a little under the weather"; "I have felt sick 
within the past week"; and "I had a cold or flu recently." An average score was calculated using the four statements, where higher scores indicate more recent illness $(\alpha=.86)$.

Medical History Form. Participants were given a medical history form about themselves and their family adapted from the West Virginia University's $4 \mathrm{H}$ health history form. The form included questions regarding allergies, medical conditions (e.g., respiratory, cardiovascular), immunizations, and recent medical events (e.g., being hospitalized). Participants indicated (yes or no) if they or any of their family members ${ }^{1}$ had a history of each medical condition. The items were divided into infectious diseases (e.g., chickenpox) and non-infectious diseases (e.g., diabetes). For each category, the items that were indicated as "yes" were summed for a composite measure. Higher numbers indicate greater incidence of infectious and non-infectious disease, respectively.

\section{Perceived Vulnerability to Disease Questionnaire (PVD; Duncan, Schaller, \&}

Park, 2009). This 15-item measure was used to assess individual differences in perceived vulnerability to disease. The questionnaire consists of two subscales: Germ Aversion and Perceived Infectability. The Germ Aversion subscale includes eight items measuring the discomfort of an individual in situations that imply high likelihood of pathogen transmission (e.g., "It really bothers me when people sneeze without covering their mouths"; $\alpha=.74$ ). The Perceived Infectability subscale consists of seven items assessing perceived personal susceptibility to disease and illness (e.g., "I am more likely than the people around me to catch an infectious disease"; $\alpha=.92$ ). All ratings were made on 7-point scale ranging from 1 ("Strongly Disagree") to 7 ("Strongly Agree"). A composite variable for each subscale was created by computing the average score across the items. Higher scores reflect greater germ aversion or perceived infectability.

\footnotetext{
${ }^{1}$ The measure for the "Family Health History" could not be computed due to a mistake during the data collection phase.
} 
Three Domain Disgust Scale (TDDS; Tybur, Lieberman, \& Griskevicius, 2009).

This 21-item measure consists of three subscales for assessing different domains of disgust: pathogen, sexual, and moral. The pathogen disgust sensitivity subscale directly pertains to disease avoidance, and serves as the most valid element of the TDDS to test BIS hypotheses (Tybur, Frankenhuis, \& Pollet, 2014). Therefore, only the pathogen disgust sensitivity subscale was included in the analyses. The pathogen disgust subscale consists of seven items (e.g. "stepping on dog poop"), and participants rated how disgusting they found each item on a 7-point scale from 0 ("not disgusting at all”) to 6 ("extremely disgusting”). A composite variable was created by computing the average score across the items $(\alpha=.78)$. Higher scores reflect greater pathogen disgust sensitivity.

\section{Results}

Means, standard deviations, and bivariate correlations among age, the BIS sensitivity indicators, recent illness index, infectious disease history, and non-infectious disease history are reported in Table 1. Age was negatively correlated with perceived infectability and pathogen disgust sensitivity, where as it was positively correlated with infectious disease history. The three BIS sensitivity indicators were positively correlated with each other. Greater recent illness was correlated with a higher perception of infectability and having experienced more non-infectious diseases was related to higher levels of perceived infectability and more germ aversion. Infectious disease history was not significantly related to any of the BIS sensitivity indicators. 
Table 1

Means, Standard Deviations, Range and Bivariate Correlations among Study 1 Measures

\begin{tabular}{|c|c|c|c|c|c|c|c|}
\hline Variables & Age & $\begin{array}{c}\text { Perceived } \\
\text { Infectability }\end{array}$ & $\begin{array}{c}\text { Germ } \\
\text { Aversion }\end{array}$ & $\begin{array}{l}\text { Pathogen } \\
\text { Disgust }\end{array}$ & $\begin{array}{l}\text { Recent } \\
\text { Illness }\end{array}$ & $\begin{array}{l}\text { Infectious } \\
\text { Disease } \\
\text { History }\end{array}$ & $\begin{array}{c}\text { Non-infectious } \\
\text { Disease } \\
\text { History }\end{array}$ \\
\hline$M$ & 19.74 & 3.62 & 4.11 & 4.83 & 2.90 & .22 & 2.63 \\
\hline$S D$ & 1.86 & 1.36 & 1.03 & 1.06 & 1.57 & .49 & 1.72 \\
\hline Range & $18-35$ & $1.00-7.00$ & $1.25-7.00$ & $1.71-7.00$ & $1.00-7.00$ & $0-3.00$ & $0-8.00$ \\
\hline $\begin{array}{l}\text { Perceived } \\
\text { Infectability }\end{array}$ & $-.12 *$ & 1 & & & & & \\
\hline Germ Aversion & .03 & $.16^{* *}$ & 1 & & & & \\
\hline $\begin{array}{l}\text { Pathogen } \\
\text { Disgust } \\
\text { Sensitivity }\end{array}$ & $-.10 *$ & $.12 *$ & $.35 * *$ & 1 & & & \\
\hline Recent Illness & .04 & $.27 * *$ & .05 & -.02 & 1 & & \\
\hline $\begin{array}{l}\text { Infectious } \\
\text { Disease } \\
\text { History }\end{array}$ & $.24 * *$ & -.03 & .01 & -.07 & .08 & 1 & \\
\hline $\begin{array}{l}\text { Non-infectious } \\
\text { Disease } \\
\text { History }\end{array}$ & -.09 & $.23 * *$ & $.11 *$ & .06 & $.24 * *$ & -.01 & 1 \\
\hline
\end{tabular}

Notes. ${ }^{*} p<.05, * * p<.01$.

The continuous BIS sensitivity measures and the health history measures split by gender and the categorical recent illness item, as well as the group comparisons from the independent samples t-tests are presented in Table 2. Participants in the recently ill group had higher levels of perceived infectability, reported more recent illness (continuous composite score), and reported a worse non-infectious disease history than the participants who were not recently ill. Women reported higher levels of perceived infectability, greater pathogen disgust sensitivity, more recent illness, and a worse non-infectious disease history than men. 
Table 2

Descriptive Statistics for BIS Sensitivity Measures and Health History Measures Split by Categorical Recent Illness and Gender.

\begin{tabular}{|c|c|c|c|c|c|c|}
\hline Variables & $\begin{array}{c}\text { Recently Ill } \\
M(S D) \\
n=99\end{array}$ & $\begin{array}{l}\text { Not Recently Ill } \\
\qquad \begin{array}{l}M(S D) \\
n=289\end{array}\end{array}$ & $\begin{array}{c}\text { Group } \\
\text { Comparison }\end{array}$ & $\begin{array}{c}\text { Men } \\
M(S D) \\
n=80\end{array}$ & $\begin{array}{l}\text { Women } \\
M(S D) \\
n=306\end{array}$ & $\begin{array}{c}\text { Group } \\
\text { Comparison }\end{array}$ \\
\hline $\begin{array}{l}\text { Perceived } \\
\text { Infectability }\end{array}$ & $3.91(1.26)$ & $3.51(1.38)$ & $t(386)=2.53 *$ & $\begin{array}{c}2.90 \\
(1.15)\end{array}$ & $\begin{array}{c}3.80 \\
(1.35)\end{array}$ & $t(384)=-5.48^{*}$ \\
\hline Germ Aversion & $4.03(.93)$ & $4.13(1.07)$ & $t(386)=-.84$ & $\begin{array}{c}4.02 \\
(1.02)\end{array}$ & $\begin{array}{c}4.13 \\
(1.02)\end{array}$ & $t(384)=-.89$ \\
\hline $\begin{array}{l}\text { Pathogen } \\
\text { Disgust } \\
\text { Sensitivity }\end{array}$ & $4.88(1.05)$ & $4.81(1.06)$ & $t(386)=.63$ & $\begin{array}{c}4.19 \\
(1.19)\end{array}$ & $\begin{array}{c}(4.99) \\
(.95)\end{array}$ & $t(384)=-6.27 * *$ \\
\hline Recent Illness & $4.32(1.49)$ & $2.41(1.28)$ & $\begin{array}{l}t(386)= \\
12.25^{* *}\end{array}$ & $\begin{array}{c}2.58 \\
(1.48)\end{array}$ & $\begin{array}{c}2.99 \\
(1.59)\end{array}$ & $t(384)=-2.09 *$ \\
\hline $\begin{array}{l}\text { Infectious } \\
\text { Disease History }\end{array}$ & $.23(.53)$ & $.21(.48)$ & $t(383)=.39$ & $.23(.53)$ & $.21(.48)$ & $t(381)=.22$ \\
\hline $\begin{array}{l}\text { Non-infectious } \\
\text { Disease History }\end{array}$ & 3.09 (1.89) & $2.48(1.64)$ & $t(374)=2.97 * *$ & $\begin{array}{c}1.97 \\
(1.55)\end{array}$ & $\begin{array}{c}2.39 \\
(1.73)\end{array}$ & $t(372)=-3.78 * *$ \\
\hline
\end{tabular}

Note. ${ }^{*} p<.05, * * p<.01$

To determine the predictive values of recent illness and health history measures on BIS sensitivity measures, a series of hierarchical linear regression analyses were conducted. Further, the possible influence of gender was explored. Age was entered into the first step of the regression models as a covariate, given the significant correlations of age with perceived infectability, pathogen disgust sensitivity, and infectious disease history. The recent illness composite variable, infectious disease history, non-infectious disease history, and gender $(1=$ Man, 2 = Woman) were entered into the second step of the regression models. In these analyses the composite recent illness variable was chosen over the dichotomous recent illness variable, because it was compromised of four different questions and was a continuous variable. Therefore, it provided more variability and was a more reliable measure. Finally, the interaction terms between gender and recent illness, gender and infectious disease history, 
and gender and non-infectious disease history were entered in the third step of the regression models. Before conducting the analyses to eliminate possible effects of sample size difference between genders $\left(n_{\text {Man }}=80, n_{\text {Woman }}=306\right)$, a random sample of 80 women were chosen to have equal sample sizes for each gender. Separate regression analyses were conducted for each BIS sensitivity measure (perceived infectability, germ aversion, and pathogen disgust sensitivity).

When predicting perceived infectability (see Table 3 for details), the only significant predictor was gender. Women reported higher levels of perceived infectability than men. When the same analyses were conducted using the entire sample (i.e., unequal numbers of men and women) with a bootstrapping method with 1000 drawings, younger age $(b=-.13)$, being more recently ill $(b=.22)$, and having experienced more non-infectious diseases $(b=$ .14) were significant predictors of higher perception of infectability $(p s<.01)$.

Table 3

Hierarchical Regression Analyses Predicting Perceived Infectability from Gender, Recent Illness, and Health History Controlling for Age

\begin{tabular}{l|ccccc}
\hline \multicolumn{1}{c|}{ Variables } & Adjusted R $\mathrm{R}^{2}$ & $B$ & $S E B$ & $b$ & $95 \%$ CI \\
\hline Step 1 & .00 & & & & \\
Age & & -.05 & .05 & -.09 & $-.14, .04$ \\
\hline Step 2 & $.16^{* *}$ & & & & \\
Gender & & .94 & .21 & $.35^{* *}$ & $.53,1.35$ \\
Recent Illness & & .13 & .07 & .14 & $-.01, .27$ \\
Infectious Disease History & & -.11 & .23 & -.04 & $-.56, .33$ \\
Non-infectious Disease History & $.17^{* *}$ & .08 & .06 & .11 & $-.04, .20$ \\
\hline Step 3 & & -.24 & .14 & -.48 & $-.52, .05$ \\
Gender x Recent Illness & & .30 & .44 & .16 & $-.56,1.17$ \\
Gender x Infectious Health History & & .19 & .13 & .47 & $-.06, .44$ \\
Gender x Non-infectious Health History & & & &
\end{tabular}

Notes. $* * p<.01$

When predicting germ aversion (see Table 4 for details), the only significant predictor was non-infectious disease history. Having experienced more non-infectious health problems was associated with higher levels of germ aversion. However, the overall model was not 
significant. When the analysis was repeated with the entire sample and bootstrapping with 1000 drawings, none of the predictors were significantly associated with germ aversion ( $p s>$ $.05)$.

Table 4

Hierarchical Regression Analyses Predicting Germ Aversion from Gender, Recent Illness, and Health History Controlling for Age

\begin{tabular}{l|ccccc}
\hline Variables & Adjusted $\mathrm{R}^{2}$ & $B$ & $S E B$ & $b$ & $95 \%$ CI \\
\hline Step 1 & -.01 & & & & \\
Age & & -.02 & .04 & .04 & $-.06, .09$ \\
\hline Step 2 & .03 & & & & \\
Gender & & -.09 & .19 & -.04 & $-.06, .09$ \\
Recent Illness & & .01 & .06 & .01 & $-.12,13$ \\
Infectious Disease History & & -.04 & .20 & -.02 & $-.44, .35$ \\
Non-infectious Disease History & .02 & .15 & .05 & $.24 * *$ & $.05, .26$ \\
\hline Step 3 & & .05 & .13 & .13 & $-.20, .30$ \\
Gender x Recent Illness & & .48 & .39 & .29 & $-.30,1.25$ \\
Gender x Infectious Disease History & & .01 & .11 & .02 & $-.22, .23$ \\
Gender x Non-infectious Disease History & & & & &
\end{tabular}

When predicting pathogen disgust sensitivity (see Table 5 for details), the only significant predictor was gender. Women reported higher levels of pathogen disgust sensitivity than men. When the analysis was repeated using the entire sample with a bootstrapping method with 1000 drawings, the only significant predictor was gender $(b=.30$; $p<.01)$ where women reported higher pathogen disgust sensitivity than men. 
Table 5

Hierarchical Regression Analyses Predicting Pathogen Disgust Sensitivity Levels from Gender, Recent Illness, and Health History Controlling for Age

\begin{tabular}{|c|c|c|c|c|c|}
\hline Variables & Adjusted $\mathrm{R}^{2}$ & $B$ & $S E B$ & $b$ & $95 \% \mathrm{CI}$ \\
\hline Step 1 & -.01 & & & & \\
\hline Age & & -.01 & .79 & -.01 & $3.16,6.29$ \\
\hline Step 2 & $.09 * *$ & & & & \\
\hline Gender & & .77 & .18 & $.33 * *$ & $.40,1.13$ \\
\hline Recent Illness & & -.03 & .06 & -.04 & $-.15, .09$ \\
\hline Infectious Disease History & & -.22 & .19 & -.09 & $-.61, .17$ \\
\hline Non-infectious Disease History & & -.11 & .05 & -.02 & $-.12, .09$ \\
\hline Step 3 & $.10 * *$ & & & & \\
\hline Gender x Recent Illness & & .15 & .13 & .36 & $-.10, .39$ \\
\hline Gender x Infectious Disease History & & .68 & .39 & .42 & $-.08,1.44$ \\
\hline Gender x Non-infectious Disease History & & -.09 & .11 & -.26 & $-.30, .13$ \\
\hline
\end{tabular}

Note. ${ }^{* *} p<.01$

\section{Discussion}

This study aimed to investigate the effect of implied activation of the physiological immune system, as assessed by illness recency and health history on BIS sensitivity (pathogen disgust sensitivity, perceived infectability, and germ aversion). Being recently ill and having experienced more non-infectious diseases were each significantly related to greater perception of infectability. Also, having experienced more non-infectious diseases was significantly related with higher levels of germ aversion. Yet, when recent illness, infectious disease history, and non-infectious disease history were simultaneous predictors of the BIS sensitivity measures, none of the illness measures were significant predictors, except non-infectious disease history on germ aversion. However, the overall model predicting germ aversion was not significant. Using a single item with a dichotomous categorization of recent illness (Miller \& Maner, 2011), it was also found that people who were recently ill reported higher levels of perceived infectability and a worse non-infectious disease history than people who were not recently ill.

Previous research has shown that women tend to have a more sensitive BIS than men 
(Duncan et al., 2009, Tybur et al., 2009). Therefore, gender was a variable of interest in this study. Consistent with the previous literature, women reported higher levels of pathogen disgust sensitivity and perceived infectability than men. However, a gender difference was not present for germ aversion. Women also reported higher levels of recent illness and noninfectious disease history than men. Gender did not moderate the relation between implied physiological immune activation and behavioral immune system sensitivity.

Overall, the findings regarding a link between BIS sensitivity and the implied activation of the physiological immune system were mixed. Perceived infectability was associated with three of the four illness measures (continuous recent illness, dichotomous recent illness, and non-infectious disease history), germ aversion was associated with one illness measure (non-infectious disease history), and pathogen disgust sensitivity was not related to any of the illness measures. These results suggest that when examining the relation between the physiological immune system and the BIS, the selection of measures for assessing each may influence the results.

Perceived infectability was most consistently related to the illness measures. Although perceived infectability is a commonly used measure in the BIS literature, it may be assessing more than just BIS sensitivity. Perceived infectability may stem not only from concerns about infectious disease, but also biological vulnerability. Further, the BIS is argued to be a mechanism that defends against infectious diseases; however, the results of this study showed that both perceived infectability and germ aversion were related with non-infectious disease history. Although they are non-infectious, these diseases (e.g. diabetes) are often also related with physiological immune system activity and they may compromise the physiological immune system, increasing susceptibility to infectious disease. Therefore, BIS sensitivity may be greater to compensate and protect against any future infectious diseases. Further research may examine this possible compensatory role that the BIS has in a body that has a 
history of non-infectious diseases.

A relation between BIS sensitivity and infectious disease history was also expected; however, it was not present. This null finding may be due to the sample and the measure that was used. To assess infectious disease history, participants indicated if they had diseases like chickenpox (see appendix A for the complete list), and many of the participants did not have any of the listed diseases that resulted in a floor effect. For most of the listed infectious diseases, effective vaccinations that provide lifetime inoculation are available, and it has been standard practice to vaccinate infants or young children for these diseases in the US for the last two decades (Cortese et al., 2008; Seward, Marin, \& Vázquez, 2008). Considering that the participants in this study were college students with a very young age ( $M=19.74$ years), it is very likely that most participants had been vaccinated for the diseases listed on the illness history form. Therefore, they did not report experiencing many infectious diseases. For future studies, using a more age diverse sample and assessing middle aged and older adults would provide more variability in infectious disease history. Also, assessment of infectious diseases that do not have a vaccine or widespread vaccine usage would be beneficial to understand the relation between infectious diseases and the BIS.

\section{Study 2}

The goal of the second study was to test the effect of BIS activation on physiological immune system activation. Participants were primed with either a disease threat or a nondisease threat. Physiological immune system activity was assessed via salivary IL-6 levels. It was expected that participants in the disease threat condition would show higher levels of IL6 compared to the participants in the non-disease threat condition. 


\section{Method}

\section{Participants}

According to a power analysis based on the effect size reported by Schaller et al. (2010; $d=0.86)$ and $\alpha=.05$, a minimum of 14 participants were required to attain $80 \%$ power to detect an effect. Replications should exceed the size of the original study $(N=28)$, and considering potential attrition or missing data a total of $47 \mathrm{WVU}$ undergraduate students participated in the study (25 female; $M_{\text {age }}=19.51$ years; $89.4 \%$ Caucasian; $93.6 \%$ heterosexual; $M d n_{\text {income }}=\$ 60,000$ - $\left.\$ 79,999\right)$. Exclusion criteria included being under 18 years of age; having a chronic or acute viral, bacterial, or immune disease; undergoing biological therapy with interleukins or interferons in the previous year; or having severe paradontopathy and acute inflammatory diseases of the oral cavity. Several participants were excluded from the primary analyses for various reasons: not showing up for the second session $(n=2)$, reporting illness during one of the sessions $(n=3)$, and undetectable IL-6 level $(n=26)$. For the primary analyses (i.e., those involving IL-6 levels), the final sample consisted of 16 participants ( 9 female, 10 in disease threat condition).

To determine if the excluded participants differed from the retained participants, statistical comparisons were made with all self-report measures and demographic items. The only significant difference was in the level of income. The excluded participants reported lower levels of income $(M=4.23, S D=2.19$, range $-\$ 19,999$ to $\$ 140,000)$ compared to the retained participants $(M=6.19, S D=2.19$, range $-\$ 19,999$ to $\$ 140,000), t(44)=2.91, p=$ .01. Further, condition comparisons were conducted for the retained participants to ensure that the groups did not differ in any demographics. There were no significant differences between the retained participants in the disease threat $(n=11)$ and the non-disease threat $(n=$ 6) conditions $(p s>.05)$ 


\section{Procedure}

Participants were recruited through the WVU Psychology Department's research participation system. A system where students can view the ongoing studies, sign up, and participate in exchange for course credits. Participants first completed an online survey, which included health related prescreening questions about recent illnesses and oral/dental problems (see Appendix B). Individuals who met the inclusion/exclusion criteria were contacted and invited to register for the main study.

The main study consisted of two sessions separated by a week to ten days $(M=7.4$ days). ${ }^{2}$ In the first session, all participants were asked to provide informed consent. If they agreed to participate, they viewed a slideshow illustrating furniture (neutral slideshow). Before and after the slideshow, participants were asked to provide saliva samples using the passive drool method. After providing their second saliva sample, participants reported their emotional state and completed the measures of disgust sensitivity and perceived vulnerability to disease. Finally, they reported their demographic information, if they had been recently ill, and if they had received a flu shot. After completing the questionnaires, participants were thanked and awarded extra credit.

In the second session, participants were randomly assigned to one of two conditions: disease threat or non-disease threat. Participants in the disease threat group viewed a slideshow portraying people with behavioral and morphological characteristics of diseases (disease slideshow); participants in the non-disease threat condition were shown a slideshow portraying people holding guns, mostly aiming towards the participants (non-disease threat

\footnotetext{
2 The study was designed as a direct replication of Schaller et al. (2010). There was one difference between the studies. In the original study, IL-6 was assessed from blood samples. In this study, IL-6 was assessed from saliva samples. IL-6 levels in blood and saliva samples have been shown to be correlated, and saliva samples are argued to be a safer and easier method to assay IL-6 levels (Williamson, Munro, Pickler, Grap, \& Elswick, 2012; Byrne et al., 2013).
} 
slideshow). Before and after the slideshow, participants provided saliva samples. After the collection of the second saliva sample, participants' self-reported emotional state was assessed, and they were asked if they had been recently ill or if they had received a flu shot. After the study was complete, participants were thanked and awarded extra credit.

\section{Materials}

Assessment of Immune Markers. Saliva samples were collected before and after each slideshow (4 samples per participant). The passive drool method was used to collect the samples. Other collection methods, such as the use of Salivettes that use cotton swabs, interfere with salivary immunoassay results for IL-6 (Minetto et al., 2007). For the passive drooling, participants were asked to pool saliva in their mouth for a few minutes and drool their saliva into a collection tube. A minimum of $2 \mathrm{ml}$ of saliva was collected for each sample. To reduce potential contamination, participants were requested to not eat, drink, use any kind of tobacco product, or brush their teeth at least two hours before the sessions. The saliva samples were checked for any contaminants before transportation to the freezer, where the samples were temporarily stored at $-20^{\circ} \mathrm{C}$ until data collection for the study was completed (September 2018 - November 2018). Each sample was aliquoted and transferred to a $-80^{\circ} \mathrm{C}$ freezer for storage until assay.

IL-6 was assayed from the saliva samples, in duplicate, using human IL-6 DuoSet ELISA kit by R\&D Systems (DY206; Minneapolis, MN), per manufacturer's protocol. The assay procedure was conducted as follows. Human IL-6 capture antibody was used to coat 96 well plates and incubated at room temperature overnight. The next day, plates were decanted and washed 3 times with wash buffer (0.05\% Tween 20 in phosphate buffered saline), and plates were blocked by adding reagent diluent ( $1 \%$ bovine serum albumin in phosphate buffered saline) and incubating for one hour at room temperature. The wash procedure was repeated, and the samples were added and incubated for 2 hours in room temperature. 
Samples were thawed, vortexed, and centrifuged at $1500 \mathrm{x}$ g for $15 \mathrm{~min}$ to separate mucus. Then, the plates were washed, detection antibody was added and incubated at room temperature overnight. With this procedure IL-6 is "sandwiched" between the capture and detection antibodies. After this process the plates were washed and Streptavidin-HRP (Horseradish peroxidase) was added. The plates were incubated for 20 minutes in room temperature. Then, the plates were washed, substrate solution (1:1 mixture of $\mathrm{H} 2 \mathrm{O} 2$ and Tetramethylbenzidine) was added, and plates were incubated for 20 minutes. The color reaction was halted by addition of stop solution $\left(2 \mathrm{~N} \mathrm{H}_{2} \mathrm{SO}_{4}\right)$. After the assay procedure was completed optical densities of the wells were determined using V max kinetic microplate reader (Molecular Devices, Sunnyvale, CA) read at $450 \mathrm{~nm}$ with a correction of $560 \mathrm{~nm}$. Values for the standard samples on each plate were plotted, and the optical densities of the unknown samples were fitted to a standard curve for each plate to determine the IL-6 level of each sample. The reported data are mean of the duplicates.

Slide-show stimuli. Three different slideshows were used: Neutral, Disease threat, and Non-disease threat. Each slideshow included 10 photographs, shown five times in a random order (see Appendix $\mathrm{C}$ for images). Each image appeared for 8 seconds, followed by a blank screen for 4 seconds before the next image appeared. The slideshows continued for 10 minutes and were presented on a flat-panel LCD monitor.

Self-reported Emotions. Subjective emotional states of the participants were assessed after each post-test saliva collection to control for possible mood changes caused by the slideshows or the saliva collection procedure. This also served as a manipulation check. Participants were shown 18 adjectives, which they rated according to how well they described their mood on a 5-point scale ranging from 0 ("not at all") to 4 ("a lot"). Composite scores were computed by taking means for fours emotional states: stressed, relaxed, scared, and disgusted. 
Perceived Stress. This 14-item measure (Cohen, Kamarck, \& Mermelstein, 1983) was used to assess stress. Participants indicated how often they experienced each item in the past month (e.g. In the last month, how often have you felt nervous and "stressed"?) on a 5point scale from 0 (“never") to 4 ("very often”). A composite variable was created by computing the average score across the items $(\alpha=.83)$. Higher scores reflect greater stress.

BIS Sensitivity Indicators. The PVD questionnaire (Duncan et al., 2009) described in Study 1 was used to assess BIS sensitivity (Perceived infectability $\alpha=.69$; Germ aversion $\alpha=.74$ ). The 32 -item Disgust Scale (Haidt, McCauley, \& Rozin, 1994) was also used to measure participants' disgust sensitivity $(\alpha=.58)$. The scale consists of eight different domains: food, animals, body products, sex, body envelope violations, death, hygiene, and magical thinking. Each domain has four items. Two of these items are answered as "true" (Disgusting) or "false" (Not disgusting). The other two are rated on a three-point scale, 0 (“Not Disgusting”), 50 (“Slightly Disgusting”), or 100 ("Very Disgusting”). A composite variable was be created by calculating the average of two scores; one score is the sum of the true/false items and the other score is the sum of the three-point scale items. Higher scores reflected greater disgust sensitivity.

\section{Results}

\section{Manipulation Check}

To determine whether the manipulation was effective, a 2 (Session: 1 and 2) x 2 (Condition: Disease threat or Non-disease threat) repeated measures ANOVA was conducted using all participants $(N=47)$ and the subjective disgust item as the dependent variable. Session was a within-subjects variable, and condition was a between-subjects variable. There was a significant main effect of Session $(F(1,37)=20.11, p<.01)$, such that participants reported feeling more disgust at the second session $(M=1.99)$ than the first session $(M=$ 1.22). There was also a significant main effect of condition $(F(1,37)=10.39, p<.01)$, such 
that participants in the disease threat condition reported feeling more disgust $(M=1.92)$ than those in the non-disease threat condition $(M=1.28)$. However, these main effects were qualified by a significant interaction between session and condition $(F(1,37)=10.46, p<.01)$ (see Figure 1). To decompose the interaction, the simple main effects were examined. The two conditions did not differ at the first session $(F(1,37)=.37, p=.55)$, but a significant difference between conditions was present at the second session $(F(1,37)=12.47, p<.01)$. Those in the disease threat condition reported more disgust than those in the non-disease threat condition at the second session. For those in the non-disease threat condition, their ratings of disgust did not differ between the first and the second sessions $(F(1,37)=.76, p=$ .39), but there was a significant difference between the two sessions for the disease threat condition $(F(1,37)=30.57, p<.01)$. Participants in the disease threat condition reported more disgust at the second session than they did at the first session. These results suggest that the manipulation worked.

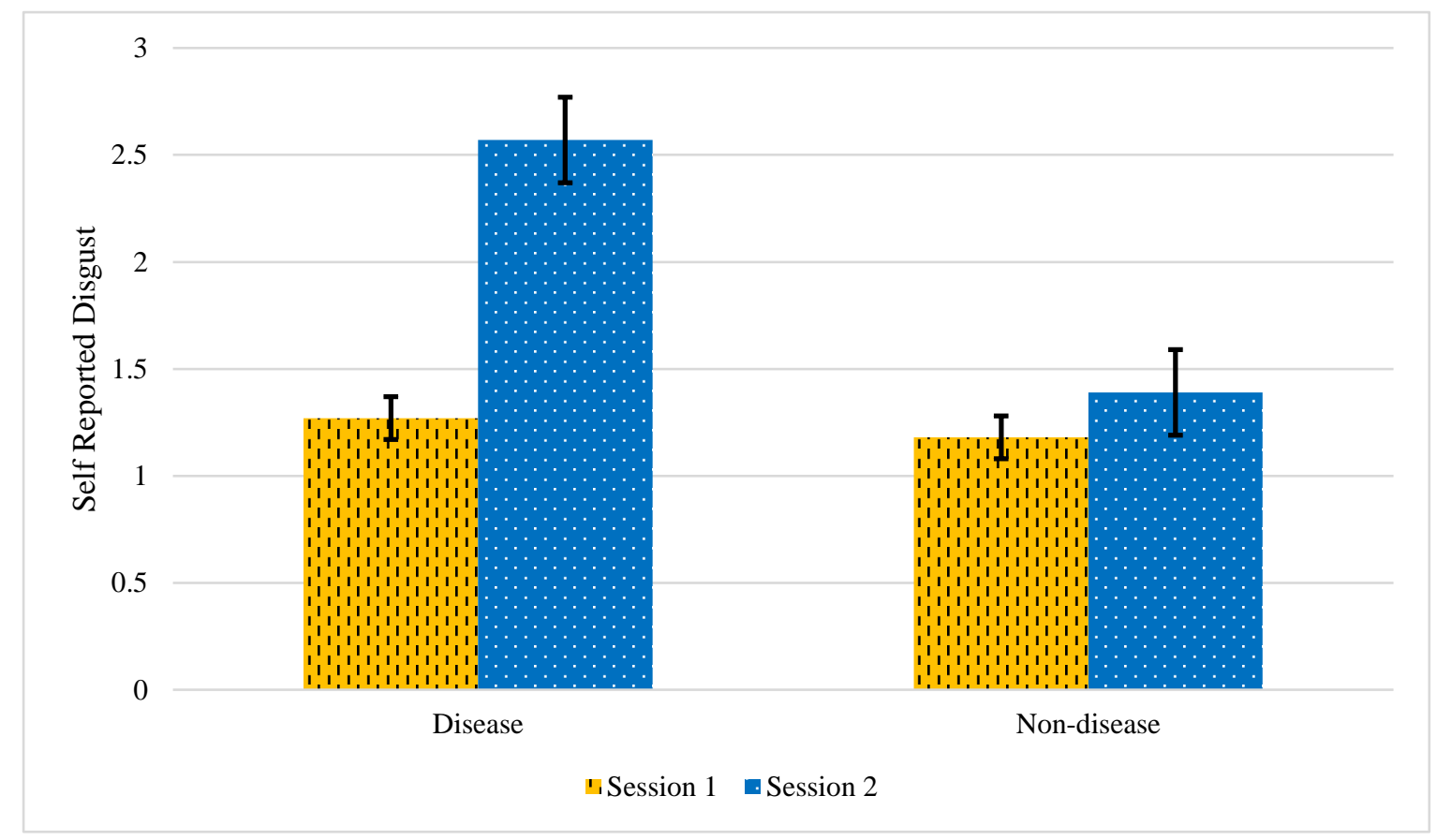

Figure 1. Mean values of the reported disgust emotion split by session and condition. 
Similar repeated measures ANOVAs were also conducted for the other self-reported emotions: relaxed, stressed, and scared. A significant main effect of Session was found for all three of the emotions (see Table 6 for means and standard deviations). Compared to the first session, participants felt less relaxed $\left(M_{\text {Session } 1}=3.52, M_{\text {Session } 2}=2.65, F(1,37)=20.35, p<\right.$ $.01)$, more stressed $\left(M_{\text {Session } 1}=1.91, M_{\text {Session } 2}=2.45, F(1,37)=8.33, p=.01\right)$, and more scared $\left(M_{\text {Session } 1}=1.07, M_{\text {Session } 2}=1.38, F(1,37)=6.52, p=.02\right)$ at the second session. There were no significant main effects of condition or interactions for the three emotions $(p s>.05)$. These results suggest that the effect of the manipulation was specific to disgust.

Table 6

Means and standard deviations of self-reported emotions split by session and condition.

\begin{tabular}{llcccc}
\hline Session & Condition & Disgusted & Relaxed & Stressed & Scared \\
\hline Session 1 & Disease & $1.27(.58)$ & $3.58(1.11)$ & $1.65(.92)$ & $1.10(.33)$ \\
\cline { 2 - 6 } & Non-Disease & $1.17(.32)$ & $3.46(1.11)$ & $2.17(.96)$ & $1.04(.11)$ \\
\hline Session 2 & Disease & $2.57(1.32)$ & $2.62(1.11)$ & $2.25(.89)$ & $1.40(.74)$ \\
\cline { 2 - 6 } & Non-Disease & $1.39(.64)$ & $2.68(.88)$ & $2.65(1.21)$ & $1.35(.69)$ \\
\hline
\end{tabular}

\section{Descriptive Statistics}

Means, standard deviations, and bivariate correlations among BIS sensitivity indicators and pre-post IL-6 levels from the two sessions are reported in Table 7. For the correlations within the BIS indicators, the entire sample was used $(N=47)$. For the correlations with the IL-6 levels, only the retained sample $(n=16)$ was used. The results show that session 1 pre-post IL-6 levels and session 2 pre-post IL-6 levels were highly correlated with each other. None of the other correlations were significant. The pre IL-6 levels from the first session were examined to see if the people in the two conditions showed differences in their baseline IL-6 levels. A significant difference was not found between the participants in the disease threat and the non-disease threat conditions $(t(14)=1.17, p=.26)$. 
Table 7

Means, Standard Deviations, Range, and Bivariate Correlations among Measures

\begin{tabular}{|c|c|c|c|c|c|c|c|c|}
\hline Variables & $\begin{array}{c}\text { Perceived } \\
\text { Infectability }\end{array}$ & $\begin{array}{c}\text { Germ } \\
\text { Aversion }\end{array}$ & $\begin{array}{c}\text { Disgust } \\
\text { Sensitivity }\end{array}$ & $\begin{array}{l}\text { Perceived } \\
\text { Stress }\end{array}$ & $\begin{array}{l}\text { Session } 1 \\
\text { Pre IL-6 }\end{array}$ & $\begin{array}{l}\text { Session } 1 \\
\text { Post IL-6 }\end{array}$ & $\begin{array}{c}\text { Session } 2 \\
\text { Pre IL-6 }\end{array}$ & $\begin{array}{l}\text { Session } 2 \\
\text { Post IL-6 }\end{array}$ \\
\hline$N(n)$ & 47 & 47 & 47 & 47 & 16 & 16 & 16 & 16 \\
\hline$M$ & 3.04 & 4.17 & 29.34 & 2.69 & 27.83 & 23.75 & 25.18 & 23.90 \\
\hline$S D$ & 1.24 & 1.11 & 2.56 & .68 & 28.25 & 21.52 & 29.34 & 27.28 \\
\hline Range & $\begin{array}{l}1.00- \\
6.14\end{array}$ & $\begin{array}{c}1.88- \\
6.50\end{array}$ & $\begin{array}{c}22.50- \\
35.00\end{array}$ & $\begin{array}{l}1.50- \\
4.10\end{array}$ & $\begin{array}{l}5.85- \\
92.85\end{array}$ & $\begin{array}{l}5.41- \\
73.44\end{array}$ & $\begin{array}{c}2.47- \\
123.90\end{array}$ & $\begin{array}{c}2.09- \\
102.23\end{array}$ \\
\hline Germ Aversion & .02 & 1 & & & & & & \\
\hline Disgust Sensitivity & -.08 & .28 & 1 & & & & & \\
\hline Perceived Stress & $.35^{*}$ & .02 & -.08 & 1 & & & & \\
\hline Session 1 Pre IL-6 & .21 & .10 & .07 & -.39 & 1 & & & \\
\hline Session 1 Post IL-6 & .26 & .09 & .04 & -.44 & $.96 * *$ & 1 & & \\
\hline Session 2 Pre IL-6 & -.12 & -.11 & .09 & -.35 & .36 & .27 & 1 & \\
\hline Session 2 Post IL-6 & -.07 & -.14 & .11 & -.30 & .47 & .35 & $.93^{* *}$ & 1 \\
\hline
\end{tabular}

Note. ${ }^{*} p<.05, * * p<.01$

\section{Primary Analyses}

To determine whether disease threat affected IL-6 levels, a 2 (Time: Pre- and Postslideshow) x 2 (Condition: Disease threat or Non-disease threat) mixed ANCOVA was performed with IL-6 levels. Time was a within-subjects variable, and condition was a between-subjects variable. To account for individual differences in naturally occurring change in IL-6 levels, a difference score between IL-6 levels after the neutral slideshow during the first session minus IL-6 levels before the neutral slideshow during the first session was calculated and entered as a covariate. There were no significant main effects or 
interaction (see Table 8).

Table 8

ANCOVA for the change in IL-6 levels $(n=16)$ by condition, time, and their interaction.

\begin{tabular}{|c|c|c|c|c|}
\hline & & $M$ & $F$ & $p$ \\
\hline \multicolumn{2}{|l|}{ Condition } & & .40 & .84 \\
\hline \multicolumn{2}{|l|}{ Disease } & 23.54 & & \\
\hline \multicolumn{2}{|l|}{ Non-disease } & 26.21 & & \\
\hline \multicolumn{2}{|l|}{ Time } & & 1.15 & .30 \\
\hline \multicolumn{2}{|l|}{ Pre } & 25.92 & & \\
\hline \multicolumn{2}{|l|}{ Post } & 23.84 & & \\
\hline \multicolumn{2}{|c|}{ Condition $x$ Time } & & 1.43 & .25 \\
\hline \multirow[t]{2}{*}{ Disease } & Pre & 24.17 & & \\
\hline & Post & 25.49 & & \\
\hline \multirow[t]{2}{*}{ Non-disease } & Pre & 26.87 & & \\
\hline & Post & 21.25 & & \\
\hline
\end{tabular}

To explore possible moderation due to individual differences in BIS sensitivity, three separate hierarchical linear regression analyses were conducted, one model for each BIS sensitivity measure (germ aversion, perceived infectability, and disgust sensitivity). In the first step of the regression models, Session 2 pre-slideshow IL-6 levels, condition, and BIS sensitivity measure (one for each regression analysis) were included. In the second step, the interaction of condition and BIS sensitivity measure was added. Session 2 post-slideshow IL6 level was entered as the outcome variable (see Table 9). Pre-slideshow IL-6 levels predicted post-slideshow IL-6 levels, but condition, BIS sensitivity, or their interaction term were not significantly related to post-slideshow IL-6 levels ${ }^{3}$.

\footnotetext{
${ }^{3}$ The same analyses were also done controlling for perceived stress levels. The results remained the same.
} 
Table 9

Three Hierarchical Regression Analyses Predicting Post IL-6 level from experimental condition and each BIS sensitivity measure

\begin{tabular}{|c|c|c|c|c|c|}
\hline Variables & Adjusted $\mathrm{R}^{2}$ & $B$ & $S E B$ & $b$ & $95 \% \mathrm{CI}$ \\
\hline PVD-PI Step 1 & $.86 * *$ & & & & \\
\hline Pre IL-6 & & .88 & .09 & $.94 * *$ & $.68,1.07$ \\
\hline Condition & & -6.36 & 5.45 & -.17 & $-18.23,5.52$ \\
\hline Perceived Infectability & & .36 & 2.05 & .02 & $-4.11,4.84$ \\
\hline PVD-PI Step 2 & $.86 * *$ & & & & \\
\hline Condition x Perceived & & 4.27 & 4.94 & .48 & $-6.61,15.14$ \\
\hline PVD-G Step 1 & $.86^{* *}$ & & & & \\
\hline Pre IL-6 & & .88 & .09 & $.94 * *$ & $.68,1.07$ \\
\hline Condition & & -6.75 & 5.68 & -.12 & $-19.13,5.63$ \\
\hline Germ Aversion & & .19 & 2.68 & .01 & $-5.68,6.05$ \\
\hline PVD-G Step 2 & $.85^{* *}$ & & & & \\
\hline Condition x Germ Aversion & & -3.94 & 5.56 & -.56 & $-16.18,8.31$ \\
\hline Disgust Step 1 & $.87 * *$ & & & & \\
\hline Pre IL-6 & & .87 & .09 & $.93 * *$ & $.68,1.06$ \\
\hline Condition & & -8.21 & 5.25 & -.15 & $-20.25,3.83$ \\
\hline Disgust Sensitivity & & .76 & .95 & .08 & $-1.31,2.82$ \\
\hline Disgust Step 2 & $.86^{* *}$ & & & & \\
\hline Condition x Disgust Sensitivity & & -.79 & 2.01 & -.53 & $-5.22,3.65$ \\
\hline
\end{tabular}

\section{Discussion}

In this study, the possible influence of BIS on the physiological immune system was tested. It was hypothesized that activation of BIS through disease related images would result in an increase of immune system marker IL-6, representing the activation of the physiological immune system. It was also hypothesized that an increase in IL-6 levels would be present in the disease threat condition but not the non-disease threat condition. According to the data, neither of the hypotheses were supported. Further, possible moderation by individual differences in BIS sensitivity was also tested, but did not show any significant results. The manipulation check indicates that the disease threat manipulation was effective. Participants who watched the disease threat slideshow reported feeling more disgust than those who watched the non-disease threat slideshow. Furthermore, the effect of the 
manipulation was specific to disgust. The conditions did not differ in other negative emotions (i.e., stressed, scared) or feelings of relaxation. Despite the self-reported effectiveness of the manipulation, the disease threat manipulation did not affect IL-6 levels, contrary to Schaller et al.'s (2010) findings. A primary reason for these null findings may be the lack of power in the sample. The ELISA kit used to assay IL-6 was not sensitive enough to detect the IL-6 levels in 25 of the participants, decreasing the analyzed sample to only 16 , which is $39 \%$ smaller than the original study by Schaller et al. (2010). Therefore, due to the reduced sample size, a possible influence of BIS on the physiological immune system may not have been detected.

This study was designed to replicate the findings of Schaller et al. (2010) using salivary IL-6 levels. In the original study, the IL-6 levels were assessed in blood samples after stimulation. Cytokines can be assessed by using two different methods. With the first method (unstimulated), the circulating levels of cytokines are examined. With the second method (stimulated), in vitro cytokine production is examined after stimulation of a sample by an immunogen (Steptoe, Hamer, \& Chida. 2007). In the study by Schaller et al. (2010), the blood samples were assessed after stimulation with a bacterial stimulus (lipopolysaccharide) that increases IL-6 secretion (Shacter, Arzadon, \& Williams, 1993), which was not the case for the present study. A possible reason for the inconsistent finding across the studies may be that BIS activation is not related to circulating cytokine levels, rather BIS activation may prime the circulating immune cells to have an increased immune response when stimulated. Indeed, an acute stressor (i.e. public speaking task) has been shown to increase stimulated levels of IL-1 $\beta$, IL-6, and TNF-a, but not unstimulated levels (Prather et al., 2009). This was argued as priming of immune cells for increased levels of pro-inflammatory cytokine production (Prather et al., 2009). Accordingly, BIS priming in this study could have primed the immune cells for more IL-6 production, but this could have been missed due to 
assessment of IL-6 from unstimulated saliva samples rather than stimulated blood samples.

As another reason for the null finding, there may not be an effect of BIS on the physiological immune system at all, or there may be other variables that are nor accounted for in this study. Previous studies have shown inconsistencies between their findings, where a main effect of BIS on physiological immune system was present in one study (Stevenson et al., 2011) and in another study this effect was dependent on disgust sensitivity level (Stevenson et al., 2012). The possible moderating effect of individual differences was tested in this study but was not present, which again may be due to a lack of power. Therefore, according to the present results, there might be no effect of BIS on the physiological immune system or the effect may be dependent on third variables that were not taken into account in the design of this study. It should also be noted that to avoid the problems faced in this study when assessing IL-6 in saliva future studies should use more sensitive kits than $9.38 \mathrm{pg} / \mathrm{mL}$, preferably operation of a quantikine ELISA kit would be suited for a more powerful assessment of IL-6.

\section{General Discussion}

Research based on the BIS theory has been increasing significantly over the past decade. But, the physiological basis of the theory, and BIS as a disease avoidance system has not been thoroughly explored. The goal of this thesis was to test if the BIS and physiological immune system were linked, and help in understanding the BIS as a system that evolved to serve a disease protecting function. Two studies were conducted to investigate 1) the relation between implied activation of the physiological immune system and BIS sensitivity, and 2) the effect of BIS activation on the physiological immune system. The first study showed that although there were several significant relations between some of the BIS and physiological immune system measures, a more general and robust relation between the two systems was not present. In the second study a significant effect of BIS activation on physiological 
immune system activation was not found.

According to previous research and our hypothesis, it was expected that greater implied activation of the physiological immune system would be related to more BIS sensitivity. The BIS is proposed to guard the individual from potential infectious diseases. When there are more threats around or there is a history of threats, the need for protection increases. Therefore, infectious disease history in particular was expected to be related to BIS sensitivity. However, the measure of infectious disease history was not related to any of the BIS sensitivity measures. Being recently ill was positively related with perception of infectability, and non-infectious disease history positively related with perceived infectability and germ aversion.

The positive relation between non-infectious disease history and perceived vulnerability to disease (perceived infectability and germ aversion) may be due to a prophylactic function of the BIS, where the body increases BIS sensitivity to avoid further contact with other pathogens while the body is compromised or susceptible to infection. Physiological immune system defends the body against infectious and non-infectious diseases, and occurrence of diseases damage the physiological immune system. A damaged or diminished physiological immune system is more likely to be compromised towards new infections. Accordingly, to avoid new infectious diseases, the perception of vulnerability to disease may be increasing to avoid any new infections to the already damaged body.

Recent illness was only related to perceived infectability, not with germ aversion or pathogen disgust sensitivity. This specific relation to perceived infectability and not the other BIS indicators raises questions of response biases specific to perceived infectability that may be playing a role in this relation. Self-report measures are prone to misleading information due to the individuals' perceptions. Considering that participants in this study reported recent illnesses according to their self-perceptions and not according to an objective measure, 
people who perceive themselves more open to infections could have reported more recent illnesses, which resulted in a significant correlation between the variables. Further, recency could have played a role on the possible effect of recent illness on perceived infectability. Perceived infectability measure consists of items like "I am more likely than the people around me to catch an infectious disease." A recently ill person may have responded to this item more favorably due to recency, although their general perceived infectability may be lower. An unbiased relation between illness recency and perceived infectability may be present, but considering the illness recency is only related with perceived infectability the possible influences of response biases should be taken into account when interpreting these findings.

Pathogen disgust sensitivity and implied activation of the physiological immune system are not related according to the results. This may be a result of the nature of disgust. Although disgust may be an evolutionarily adaptive emotion, it is also in some part learned. Research indicates that disgust responses can be learned through evaluative conditioning. Giving a disgust reaction to previously neutral stimuli can result in labeling the stimuli as disgusting (Olatunji, Forsyth, \& Cherian, 2007). Social learning is another way that individuals learn to associate different stimuli with disgust (Curtis, Barra, \& Aunger, 2011). Social learning helps humans especially in early development to identify potential threats in their environment. As such, parents have a strong influence on their offsprings' disgust reactions. Understanding the concept of disgust takes time and reaches its development around the age of 7 (Rozin, Fallon, \& Augustoni-Ziskind, 1986), and people show resemblance with their parents in their disgust sensitivity (Davey, Forster, \& Mayhew, 1993; Rozin, Fallon, \& Mandell, 1984). Therefore, the lack of relation between health history and disgust sensitivity may be because disgust does not only serve a disease avoidance function. It is also in part a socioculturally derived, or learned, response. 
According to the BIS theory, the physiological and the behavioral immune systems are expected to work in cohesion (Ackerman et al., 2018). However, the findings of this thesis did not support the assumptions. The relations between health history and BIS sensitivity was minimal, and increasing BIS activity did not have an effect on physiological immune system activity. The latter finding should be interpreted with caution, given the small sample size and issues with IL-6 detection. Overall, these results contradict the fundamental assumptions of the BIS theory and many of the BIS studies. This raises concerns about the development of the BIS theory and assumptions underlying much of the existing literature.

Since the debut of the theory (Schaller, 2006), the study of the BIS has grown substantially. A search of the term "Behavioral Immune System" in PsycINFO resulted in 56 published papers between 2007 and 2018, with the majority $(n=45,80 \%)$ of papers from the last five years (i.e., 2013-2018). Moreover, most of those papers $(n=39,70 \%)$ focused on the social by-products of the BIS. BIS research has grown rapidly without systematic, empirical testing of the original theory, its assumptions, and appropriate methods. In a recent review of the BIS literature, it was noted that one of the fundamental assumptions of the BIS theory, i.e., the connection between the BIS and the physiological immune system, had received relatively little empirical attention (Ackerman et al., 2018). The aim of this thesis was to examine this assumption of the theory, and the results seem to challenge the assumptions of the BIS theory.

Deriving from the findings of this research, future studies could help in making the BIS theory better. Perceived Vulnerability to Disease questionnaire by Duncan et al. (2009), is one of the most frequently used measures for assessing BIS sensitivity and is considered to assess trait-level BIS sensitivity (Tybur et al., 2014). According to the findings of Study 1, the widely used perceived infectability subscale of the PVD questionnaire may be showing state level functions or could be prone to response biases. Studying the relation between 
recent illnesses by using objective measures of illness and conducting a longitudinal study would help in a better understanding about the possible shortcomings of this measure. Resolving these possible issues would strengthen BIS methodology.

Although the study of BIS is relatively new, the study of disgust, an essential part of the BIS, has a decades long history. The literature from the 1980s provides support for the possible place of learning in disgust. However, the role of learning has not been incorporated into BIS theory. How learning and experiences influence our evolutionary mechanisms and consequently BIS should be questioned and studied thoroughly for a more complete understanding of the BIS.

As with the BIS theory, the studies of this thesis had limitations. The examination of the influence of physiological immune system history on BIS was done in a correlational design and a causal inference cannot be made. Examination of this relation with a longitudinal design is essential to understand the possible effects of illnesses on the BIS measures that are considered as traits. Especially assessing different immune markers in different time points and examining their interaction with BIS measures would help in making the relation between the systems clearer. However, weight should be given to choosing of the assays. As it can be seen from the second study, not having a sensitive assay can result in an underpowered sample and come up as a limitation. Future studies with more sensitive and more diverse assays of different immune markers would help in understanding the relation between BIS and the physiological immune system. Another limitation of this study is the operation of self-report measures to assess recent illness and health history. Using a self-report measure for health variables is prone to biases and could be influenced by individual differences. Future studies using objective health measures for assessing health history and recent illnesses would help in avoiding these biases in measurement.

On the whole, this thesis aimed to examine the assumed relation between the BIS and 
the physiological immune system. These findings did not support the assumption that the BIS and the physiological immune system were associated. The theory of the BIS should be understood and developed more thoroughly to have a more clear and reliable research path for this vital system. More research with an established methodology and a compelling manner is obligatory to have better understanding of this system that protects us from diseases and is essential in our survival. 


\section{References}

Aarøe, L., Osmundsen, M., \& Petersen, M. B. (2016). Distrust as a disease avoidance strategy: Individual differences in disgust sensitivity regulate generalized social trust. Frontiers in psychology, 7, 1038.

Abbas, A. K., Lichtman, A. H., \& Pillai, S. (2014). Cellular and molecular immunology Ebook. Elsevier Health Sciences.

Abbas, K. A., Lichtman, A. H., \& Pober, J. S. (2000). Cellular and mollecular immunology.4th ed. Elsevier Health Sciences.

Ackerman, J. M., Becker, D. V., Mortensen, C. R., Sasaki, T., Neuberg, S. L., \& Kenrick, D. T. (2009). A pox on the mind: Disjunction of attention and memory in the processing of physical disfigurement. Journal of Experimental Social Psychology, 45(3), 478485.

Ackerman, J. M., Hill, S. E., \& Murray, D. R. (2018). The behavioral immune system: Current concerns and future directions. Social and Personality Psychology Compass.

Al-Shawaf, L., Lewis, D. M., Alley, T. R., \& Buss, D. M. (2015). Mating strategy, disgust, and food neophobia. Appetite, 85, 30-35.

Baracos, V. E., Whitmore, W. T., \& Gale, R. (1987). The metabolic cost of fever. Canadian journal of physiology and pharmacology, 65(6), 1248-1254.

Byrne, M. L., O’Brien-Simpson, N. M., Reynolds, E. C., Walsh, K. A., Laughton, K., Waloszek, J. M., ... \& Allen, N. B. (2013). Acute phase protein and cytokine levels in serum and saliva: a comparison of detectable levels and correlations in a depressed and healthy adolescent sample. Brain, Behavior, and Immunity, 34, 164-175.

Clark, J. A., \& Fessler, D. M. (2014). Recontextualizing the behavioral immune system within psychoneuroimmunology. Evolutionary Behavioral Sciences, 8(4), 235. 
Cortese, M. M., Jordan, H. T., Curns, A. T., Quinlan, P. A., Ens, K. A., Denning, P. M., \& Dayan, G. H. (2008). Mumps vaccine performance among university students during a mumps outbreak. Clinical Infectious Diseases, 46(8), 1172-1180.

Curtis, V., De Barra, M., \& Aunger, R. (2011). Disgust as an adaptive system for disease avoidance behaviour. Philosophical Transactions of the Royal Society B: Biological Sciences, 366(1563), 389-401.

Davey, G. C., Forster, L., \& Mayhew, G. (1993). Familial resemblances in disgust sensitivity and animal phobias. Behaviour Research and Therapy, 31(1), 41-50.

Delves, P. J., \& Roitt, I. M. (2000). The immune system. New England journal of medicine, 343(1), 37-49.

Diercks, G. F., \& Kluin, P. M. (2016). Basic Principles of the Immune System and Autoimmunity. In Autoimmune Bullous Diseases (pp. 3-12). Springer, Cham.

Duncan, L. A., Schaller, M., \& Park, J. H. (2009). Perceived vulnerability to disease: Development and validation of a 15-item self-report instrument. Personality and Individual differences, 47(6), 541-546.

Faulkner, J., Schaller, M., Park, J. H., \& Duncan, L. A. (2004). Evolved disease-avoidance mechanisms and contemporary xenophobic attitudes. Group Processes \& Intergroup Relations, 7(4), 333-353.

Ferguson, E., \& Daniel, E. (1995). The Illness Attitudes Scale (IAS): A psychometric evaluation on a non-clinical population. Personality and Individual Differences, 18(4), 463-469.

Fessler, D. M., Eng, S. J., \& Navarrete, C. D. (2005). Elevated disgust sensitivity in the first trimester of pregnancy: Evidence supporting the compensatory prophylaxis hypothesis. Evolution and Human Behavior, 26(4), 344-351. 
Gordon, S. (2016). Phagocytosis: an immunobiologic process. Immunity, 44(3), 463-475.

Haidt, J., McCauley, C., \& Rozin, P. (1994). Individual differences in sensitivity to disgust: A scale sampling seven domains of disgust elicitors. Personality and Individual differences, 16(5), 701-713.

Hamrick, N., Cohen, S., \& Rodriguez, M. S. (2002). Being popular can be healthy or unhealthy: stress, social network diversity, and incidence of upper respiratory infection. Health Psychology, 21(3), 294.

Heinrich, P. C., Behrmann, I., Serge, H. A. A. N., Hermanns, H. M., Müller-Newen, G., \& Schaper, F. (2003). Principles of interleukin (IL)-6-type cytokine signalling and its regulation. Biochemical journal, 374(1), 1-20.

Janeway C.A., et al. (2001). Immunobiology: The Immune System in Health and Disease. New York: Garland Science.

Kandrik, M., Hahn, A. C., Fisher, C. I., Wincenciak, J., DeBruine, L. M., \& Jones, B. C. (2017). Are physiological and behavioral immune responses negatively correlated? Evidence from hormone-linked differences in men's face preferences. Hormones and behavior, 87, 57-61.

Kawai, T., \& Akira, S. (2006). Innate immune recognition of viral infection. Nature immunology, 7(2), 131.

Kellner, R. (1986). Somatization and hypochondriasis. Praeger Publishers.

Kiecolt-Glaser, J. K., Glaser, R., Williger, D., Stout, J., Messick, G., Sheppard, S., ... \& Donnerberg, R. (1985). Psychosocial enhancement of immunocompetence in a geriatric population. Health Psychology, 4(1), 25.

Kurzban, R., \& Leary, M. R. (2001). Evolutionary origins of stigmatization: the functions of social exclusion. Psychological bulletin, 127(2), 187. 
Loh, E. H., Zambrana-Torrelio, C., Olival, K. J., Bogich, T. L., Johnson, C. K., Mazet, J. A., ... \& Daszak, P. (2015). Targeting transmission pathways for emerging zoonotic disease surveillance and control. Vector-Borne and Zoonotic Diseases, 15(7), 432437.

Mandell, G. L. (1995). Cytokines, phagocytes, and pentoxifylline. Journal of cardiovascular pharmacology, 25, S20-2.

Maier, S. F., \& Watkins, L. R. (1998). Cytokines for psychologists: implications of bidirectional immune-to-brain communication for understanding behavior, mood, and cognition. Psychological review, 105(1), 83.

Miller, G. E., \& Cohen, S. (2001). Psychological interventions and the immune system: A meta-analytic review and critique. Health Psychology, 20(1), 47.

Miller, S. L., \& Maner, J. K. (2011). Sick body, vigilant mind: The biological immune system activates the behavioral immune system. Psychological science, 22(12), 1467-1471.

Minetto, M. A., Gazzoni, M., Lanfranco, F., Baldi, M., Saba, L., Pedrola, R., ... \& Rainoldi, A. (2007). Influence of the sample collection method on salivary interleukin-6 levels in resting and post-exercise conditions. European journal of applied physiology, 101(2), 249-256.

Minetto, M., Rainoldi, A., Gazzoni, M., Terzolo, M., Borrione, P., Termine, A., ... \& Paccotti, P. (2005). Differential responses of serum and salivary interleukin-6 to acute strenuous exercise. European journal of applied physiology, 93(5-6), 679-686.

Mortensen, C. R., Becker, D. V., Ackerman, J. M., Neuberg, S. L., \& Kenrick, D. T. (2010). Infection breeds reticence: The effects of disease salience on self-perceptions of personality and behavioral avoidance tendencies. Psychological Science, 21(3), 440447. 
Murray, D. R. (2014). Direct and indirect implications of pathogen prevalence for scientific and technological innovation. Journal of Cross-Cultural Psychology, 45(6), 971-985.

Murray, D. R., Jones, D. N., \& Schaller, M. (2013). Perceived threat of infectious disease and its implications for sexual attitudes. Personality and Individual Differences, 54(1), 103-108.

Neuberg, S. L., Kenrick, D. T., \& Schaller, M. (2011). Human threat management systems: Self-protection and disease avoidance. Neuroscience \& Biobehavioral Reviews, 35(4), 1042-1051.

Olatunji, B. O., Forsyth, J. P., \& Cherian, A. (2007). Evaluative differential conditioning of disgust: A sticky form of relational learning that is resistant to extinction. Journal of Anxiety Disorders, 21(6), 820-834.

Oosterhoff, B., Shook, N. J., \& Ford, C. (2018). Is that disgust I see? Political ideology and biased visual attention. Behavioural brain research, 336, 227-235.

Park, J. H., Faulkner, J., \& Schaller, M. (2003). Evolved disease-avoidance processes and contemporary anti-social behavior: Prejudicial attitudes and avoidance of people with physical disabilities. Journal of Nonverbal behavior, 27(2), 65-87.

Prather, A. A., Carroll, J. E., Fury, J. M., McDade, K. K., Ross, D., \& Marsland, A. L. (2009). Gender differences in stimulated cytokine production following acute psychological stress. Brain, behavior, and immunity, 23(5), 622-628.

Pressman, S. D., Cohen, S., Miller, G. E., Barkin, A., Rabin, B. S., \& Treanor, J. J. (2005). Loneliness, social network size, and immune response to influenza vaccination in college freshmen. Health Psychology, 24(3), 297.

Raison, C. L., Capuron, L., \& Miller, A. H. (2006). Cytokines sing the blues: inflammation and the pathogenesis of depression. Trends in immunology, 27(1), 24-31. 
Rozin, P., Fallon, A., \& Augustoni-Ziskind, M. (1986). The child's conception of food: The development of categories of acceptable and rejected substances. Journal of Nutrition Education, 18(2), 75-81.

Rozin, P., Fallon, A., \& Mandell, R. (1984). Family resemblance in attitudes to foods. Developmental Psychology, 20(2), 309.

Seward, J. F., Marin, M., \& Vázquez, M. (2008). Varicella vaccine effectiveness in the US vaccination program: a review. The Journal of infectious diseases, 197(Supplement 2), S82-S89.

Schaller, M. (2006). Parasites, behavioral defenses, and the social psychological mechanisms through which cultures are evoked. Psychological Inquiry, 17(2), 96-101.

Schaller, M., \& Duncan, L. A. (2007). The behavioral immune system: Its evolution and social psychological implications. In J. P. Forgas, M. G. Haselton, \& W. von Hippel (Eds.), Evolution and the social mind: Evolutionary psychology and social cognition (pp. 293-307). New York: Psychology Press.

Schaller, M., \& Murray, D. R. (2008). Pathogens, personality, and culture: disease prevalence predicts worldwide variability in sociosexuality, extraversion, and openness to experience. Journal of personality and social psychology, 95(1), 212.

Schaller, M., \& Park, J. H. (2011). The behavioral immune system (and why it matters). Current Directions in Psychological Science, 20(2), 99-103.

Schaller, M., Miller, G. E., Gervais, W. M., Yager, S., \& Chen, E. (2010). Mere visual perception of other people's disease symptoms facilitates a more aggressive immune response. Psychological Science, 21(5), 649-652.

Shacter, E., Arzadon, G. K., \& Williams, J. A. (1993). Stimulation of interleukin-6 and prostaglandin E2 secretion from peritoneal macrophages by polymers of albumin. Blood, 82(9), 2853-2864. 
Steptoe, A., Hamer, M., \& Chida, Y. (2007). The effects of acute psychological stress on circulating inflammatory factors in humans: a review and meta-analysis. Brain, behavior, and immunity, 21(7), 901-912.

Stevenson, R. J., Hodgson, D., Oaten, M. J., Barouei, J., \& Case, T. I. (2011). The effect of disgust on oral immune function. Psychophysiology, 48(7), 900-907.

Stevenson, R. J., Hodgson, D., Oaten, M. J., Moussavi, M., Langberg, R., Case, T. I., \& Barouei, J. (2012). Disgust elevates core body temperature and up-regulates certain oral immune markers. Brain, behavior, and immunity, 26(7), 1160-1168.

Stevenson, R. J., Hodgson, D., Oaten, M. J., Sominsky, L., Mahmut, M., \& Case, T. I. (2015). Oral immune activation by disgust and disease-related pictures. Journal of Psychophysiology, 29(3), 119.

Terrizzi, J. A. (2017). Is religion an evolutionarily evoked disease-avoidance strategy?. Religion, Brain \& Behavior, 7(4), 328-330.

Tybur, J. M., Frankenhuis, W. E., \& Pollet, T. V. (2014). Behavioral immune system methods: Surveying the present to shape the future. Evolutionary Behavioral Sciences, 8(4), 274.

Tybur, J. M., Lieberman, D., \& Griskevicius, V. (2009). Microbes, mating, and morality: individual differences in three functional domains of disgust. Journal of personality and social psychology, 97(1), 103.

van Leeuwen, F., \& Petersen, M. B. (2017). The behavioral immune system is designed to avoid infected individuals, not outgroups. Evolution and Human Behavior.

Vyse, T. J., \& Todd, J. A. (1996). Genetic analysis of autoimmune disease. Cell, 85(3), 311318. 
Welling, L. L. M., Conway, C. A., Debruine, L. M., \& Jones, B. C. (2007). Perceived vulnerability to disease is positively related to the strength of preferences for apparent health in faces. Journal of Evolutionary Psychology, 5(1), 131-139.

Williamson, S., Munro, C., Pickler, R., Grap, M. J., \& Elswick, R. K. (2012). Comparison of biomarkers in blood and saliva in healthy adults. Nursing research and practice, 2012. 


\section{APPENDIX A- COMPLETE LIST OF MEASURES}

\section{Illness Recency (Miller \& Maner, 2011)}

Please indicate your agreement $(1=$ strongly disagree, $7=$ strongly agree $)$ with four statements:

1. Over the past couple days, I have not been feeling well".

2. Lately, I have been feeling a little under the weather.

3. I have felt sick within the past week.

4. I had a cold or flu recently.

Please indicated the last time you had a cold by selecting from among the following response options:

- today

- a couple days ago

- a week ago

- a couple weeks ago

- a month ago

- a few months ago

- a year or more ago

\section{Health History Questions}

Please give information on the medical history of you and any blood relatives. Please indicate whether you or a family member (e.g. your mother, father, sister, brother, aunt, uncle, etc.) have or previously had any of the following conditions.

1. Allergies

2. Asthma

3. Bronchitis

4. Emphysema

5. Tuberculosis

6. Cystic Fibrosis

7. Ulcers

8. Inflammatory Bowel

9. Cleft Lip or Palate

10. Other Gastrointestinal Conditions

11. High Blood Pressure

12. Heart Attack

13. Stroke

14. Congestive Heart Failure

15. Atherosclerosis

16. Heart Rhythm Abnormality

17. Congenital Heart Defect

18. Mononucleosis 
19. Hemophilia

20. Leukemia

21. Lymphomas

22. Hodgkin's Disease

23. Factor V Leiden

24. Kidney Failure

25. Dialysis/Transplant

26. Other Kidney conditions

27. Hepatitis

28. Cirrhosis

29. Other Liver Disease

30. Epilepsy

31. Hydrocephalus

32. Multiple Sclerosis

33. Huntington's Chorea

34. Seizures/Convulsions

35. Diabetes (Adult or Juvenile)

36. Thyroid (Hyper/Hypo)

37. Adrenal

38. Club Foot

39. Scoliosis

40. Arthritis (Osteo or Rheumatoid)

41. Lupus

42. Cerebral Palsy

43. Muscular Dystrophy

44. Spina Bifida

45. Blindness

46. Glaucoma

47. Cataracts

48. Deafness or other hearing problems

49. Mental illness (e.g. Depression, Bipolar, Schizophrenia)

50. Alcohol or Drug abuse

51. Eating disorders

52. Mental Retardation or Developmental Disability

General questions: Explain "Yes" answers below.

1. Had any recent injury, illness, or infectious diseases? (Ex)

2. Have a chronic or recurring illness/condition? (N/I)

3. Ever been hospitalized? (Ex)

4. Ever had surgery? (Ex)

5. Have frequent headaches? (N/I)

6. Ever had a head injury? (Ex)

7. Ever been knocked unconscious? (Ex)

8. Wear glasses, contacts or protective eye wear? (P/I)

9. Ever had frequent ear infections? (Ex)

10. Ever passed out during or after exercise? (N/I)

11. Ever been dizzy during or after exercise? (N/I)

12. Ever had seizures? (N/I)

13. Ever had chest pain during or after exercise? (N/I)

14. Ever had high blood pressure? (N/I) 
15. Ever been diagnosed with a heart murmur? (N/I)

16. Ever had back problems? (P/I)

17. Ever had problems with joints (e.g., knees, ankles)? (P/I)

18. Have an orthodontic appliance being brought to the event? (P/I)

19. Have any skin problems (e.g., itching, rash, acne)? (P/I)

20. Have diabetes? (N/I)

21. Have asthma? (N/I)

22. Had mononucleosis in the past 12 months? (I)

23. Had problems with diarrhea/constipation? (N/I)

24. Have problems with sleepwalking? (P)

25. If female, have an abnormal menstrual history? (Ex)

26. Have a history of bed-wetting? (P)

27. Ever had an eating disorder? (P)

28. Ever had emotional difficulties for which professional help was sought? (P)

29. Measles (I)

30. Chickenpox (I)

31. Mumps (I)

32. Hepatitis A (I)

33. Hepatitis B (I)

34. Hepatitis C (I)

$(\mathrm{I})=$ Infectious Disease; $(\mathrm{P})=$ Mental related problem; $(\mathrm{P} / \mathrm{I})=$ Physical Impairment; $(\mathrm{N} / \mathrm{I})=$ Non-infectious Disease; $(\mathrm{Ex})=$ Excluded

\section{Perceived Stress Scale (Cohen, Kamarck, \& Mermelstein, 1983)}

The questions in this scale ask you about your feelings and thoughts during the last month. In each case, you will be asked to indicate how often you felt or thought a certain way.

Although some of the questions are similar, there are differences between them and you should treat each one as a separate question. The best approach is to answer each question fairly quickly. That is, don't try to count up the number of times you felt a particular way, but rather indicate the alternative that seems like a reasonable estimate.

For each question choose from the following alternatives:
0 . never
1. almost never
2. sometimes
3. fairly often
4. very often

1. In the last month, how often have you been upset because of something that happened unexpectedly?

2. In the last month, how often have you felt that you were unable to control the important things in your life?

3. In the last month, how often have you felt nervous and "stressed"?

4. In the last month, how often have you dealt successfully with irritating life hassles?

5. In the last month, how often have you felt that you were effectively coping with important changes that were occurring in your life?

6. In the last month, how often have you felt confident about your ability to handle your personal problems? 
7. In the last month, how often have you felt that things were going your way?

8. In the last month, how often have you found that you could not cope with all the things that you had to do?

9. In the last month, how often have you been able to control irritations in your life?

10. In the last month, how often have you felt that you were on top of things?

11. In the last month, how often have you been angered because of things that happened that were outside of your control?

12. In the last month, how often have you found yourself thinking about things that you have to accomplish?

13. In the last month, how often have you been able to control the way you spend your time?

14. In the last month, how often have you felt difficulties were piling up so high that you could not overcome them?

\section{Perceived Vulnerability to Disease Questionnaire (Duncan, Schaller, \& Park, 2009)}

Instructions: Please indicate the extent to which you agree or disagree with each statement below using the following scale. There are no right or wrong answers. Please think about each statement carefully before answering.

1 - Strongly Disagree

2 - Disagree

3 - Slightly Disagree

4 - Neither Agree nor Disagree

5 - Slightly Agree

6 - Agree

7 - Strongly Agree

1. In general, I am very susceptible to colds, flu and other infectious diseases.

2. I am unlikely to catch a cold, flu or other illness, even if it is 'going around'.

3. If an illness is 'going around', I will get it.

4. My immune system protects me from most illnesses that other people get.

5. I am more likely than the people around me to catch an infectious disease.

6. My past experiences make me believe I am not likely to get sick even when my friends are sick.

7. I have a history of susceptibility to infectious disease.

8. I prefer to wash my hands pretty soon after shaking someone's hand.

9. I avoid using public telephones because of the risk that I may catch something from the previous user.

10. I do not like to write with a pencil someone else has obviously chewed on.

11. I dislike wearing used clothes because you do not know what the last person who wore it was like.

12. I am comfortable sharing a water bottle with a friend.

13. It really bothers me when people sneeze without covering their mouths.

14. It does not make me anxious to be around sick people.

15. My hands do not feel dirty after touching money.

Three-Domain Disgust Scale (Tybur, Lieberman, \& Griskevicius, 2009) 
The following items describe a variety of concepts. Please rate how disgusting you find the concepts described in the items, where 0 means that you do not find the concept disgusting at all and 6 means that you find the concept extremely disgusting.

1. Shoplifting a candy bar from a convenience store

2. Hearing two strangers having sex

3. Stepping on dog poop

4. Stealing from a neighbor

5. Performing oral sex

6. Sitting next to someone who has red sores on their arm

7. A student cheating to get good grades

8. Watching a pornographic video

9. Shaking hands with a stranger who has sweaty palms

10. Deceiving a friend

11. Finding out that someone you don't like has sexual fantasies about you

12. Seeing some mold on old leftovers in your refrigerator

13. Forging someone's signature on a legal document

14. Bringing someone you just met back to your room to have sex

15. Standing close to a person who has body odor

16. Cutting to the front of a line to purchase the last few tickets to a show

17. A stranger of the opposite sex intentionally rubbing your thigh in an elevator

18. Seeing a cockroach run across the floor

19. Intentionally lying during a business transaction

20. Having anal sex with someone of the opposite sex

21. Accidentally touching a person's bloody cut

\section{The Disgust Scale (Haidt, McCauley, \& Rozin, 1994)}

The following items describe a variety of concepts. Please rate how disgusting you find the concepts described in the items

(R) indicates reverse woring. In each domain. the first 2 items are personal-reaction items (scored 0.1 ) and the second 2 items are disgust-rating items (scored 0,0.5,1 1).

\section{Food}

1. I might be willing to try eating monkey meat, under some circumstances. (R)

2. It bothers me to see someone in a restaurant eating messy food with his fingers.

3. You see someone put ketchup on vanilla ice cream, and eat it.

4. You are about to drink a glass of milk when you smell that it is spoiled.

\section{Animals}

5. It would bother me to see a rat run across my path in a park.

6. Seeing a cockroach in someone else's house doesn't bother me. (R)

7. You see maggots on a piece of meat in an outdoor garbage pail.

8. You are walking barefoot on concrete, and you step on an earthworm.

\section{Body Products}

9. It bothers me to hear someone clear a throat full of mucous.

10. If I see someone vomit, it makes me sick to my stomach.

11. You see a bowel movement left unflushed in a public toilet.

12. While you are walking through a tunnel under a railroad track, you smell urine. Sex 
13. I think homosexual activities are immoral.

14. I think it is immoral for people to seek sexual pleasure from animals.

15. You hear about an adult woman who has sex with her father.

16. You hear about a 30 year old man who seeks sexual relationships with 80 year old women.

\section{Envelope Violations}

17. It would bother me to be in a science class, and to see a human hand preserved in a jar.

18. It would not upset me at all to watch a person with a glass eye take the eye out of the socket. (R)

19. You see someone accidentally stick a fishing hook through his finger.

20. You see a man with his intestines exposed after an accident.

\section{Death}

21. It would bother me tremendously to touch a dead body.

22. I would go out of my way to avoid walking through a graveyard.

23. Your friend's pet cat dies, and you have to pick up the dead body with your bare hands.

24. You accidentally touch the ashes of a person who has been cremated.

\section{Hygiene}

25. I never let any part of my body touch the toilet seat in public restrooms.

26. I probably would not go to my favorite restaurant if I found out that the cook had a cold.

27. You take a sip of soda, and then realize that you drank from the glass that an acquaintance of yours had been drinking from.

28. You discover that a friend of yours changes underwear only once a week.

\section{Magic}

29. Even if I was hungry, I would not drink a bowl of my favorite soup if it had been stirred by a used but thoroughly washed flyswatter.

30. It would bother me to sleep in a nice hotel room if I knew that a man had died of a heart attack in that room the night before.

31. A friend offers you a piece of chocolate shaped like dog-doo.

32. As part of a sex education class, you are required to inflate a new unlubricated condom, using your mouth.

\section{Subjective Emotional State (Schaller et al., 2010)}

On 5-point scales ranging from 0 to 4 , participants will rate the extent to which each of adjectives accurately described their mood. Composite measures of four specific emotional states were computed as mean ratings of 3 adjectives each: stressed (stressed, tense, overwhelmed), relaxed (relaxed, calm, at ease), scared (scared, afraid, fearful), and disgusted (disgusted, repulsed, revolted).

\section{Demographics}

Gender (circle): Male Female Transgender Other:

Age:

Marital Status (check one): 


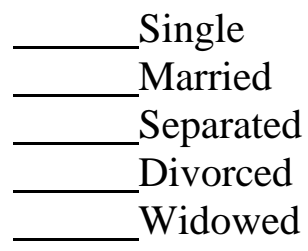

Race/Ethnicity (check all that apply):

White/Caucasian
Hispanic/Latino(a)
African-American/Black
Asian
Native American
Other - Please list:

What is your religious affiliation (check one):

Christian - Protestant
Christian - Catholic
Hindu
Buddhist
Not religious

Muslim Jewish Atheist Agnostic Other - Please list:

Please indicate your sexuality (Check one):

1) Straight/Heterosexual

2) Lesbian/Homosexual Female

3) Gay/Homosexual Male

4) Bi-sexual

5) Other:

What would you estimate your combined family income to be?
A- $0-\$ 19,999$
B- $\$ 20,000$ - $\$ 39,999$
C- $\$ 40,000$ - $\$ 59,999$
D- $\$ 60,000$ - $\$ 79,999$
E- $\$ 80,000$ - $\$ 99,999$
F- $\$ 100,000$ - $\$ 119,999$
G- $\$ 120,000$ - $\$ 139,999$
H- $\$ 140,000$ or more

How would you classify your political ideology?
EXTREMELY

How would you rate your overall health at the present time?

$1=$ excellent

$2=\operatorname{good}$

$3=$ fair

$4=$ poor 
Did you have a flu shot in the last month?

Yes/No

Did you have any recent injury, illness, or infectious diseases in the last week? If yes please indicate ...

No 


\section{APPENDIX B- SCREENING SURVEY}

Please answer the questions as "yes" or "no".

Do you have:

- Any recent injury, illness, or infectious disease?

- A chronic or recurring illness/condition?

- Frequent headaches?

- Frequent ear infections?

- Diabetes?

- Asthma?isêpi

- Any skin problems (e.g., itching, rash)? If yes please define:

- Problems with diarrhea/constipation?

- Any oral infections, or diseases?

- Any if the following; Measlesisepi, Chickenpox, German measles, Mumpsisep, Hepatitis A

- Any medications that you are currently using? If yes please define:

Did you have in the previous year:

- Exposure to biological therapies with interleukins or interferons?

- Had any oral surgeries? 
APPENDIX C- SLIDE-SHOW STIMULI

Disease Threat
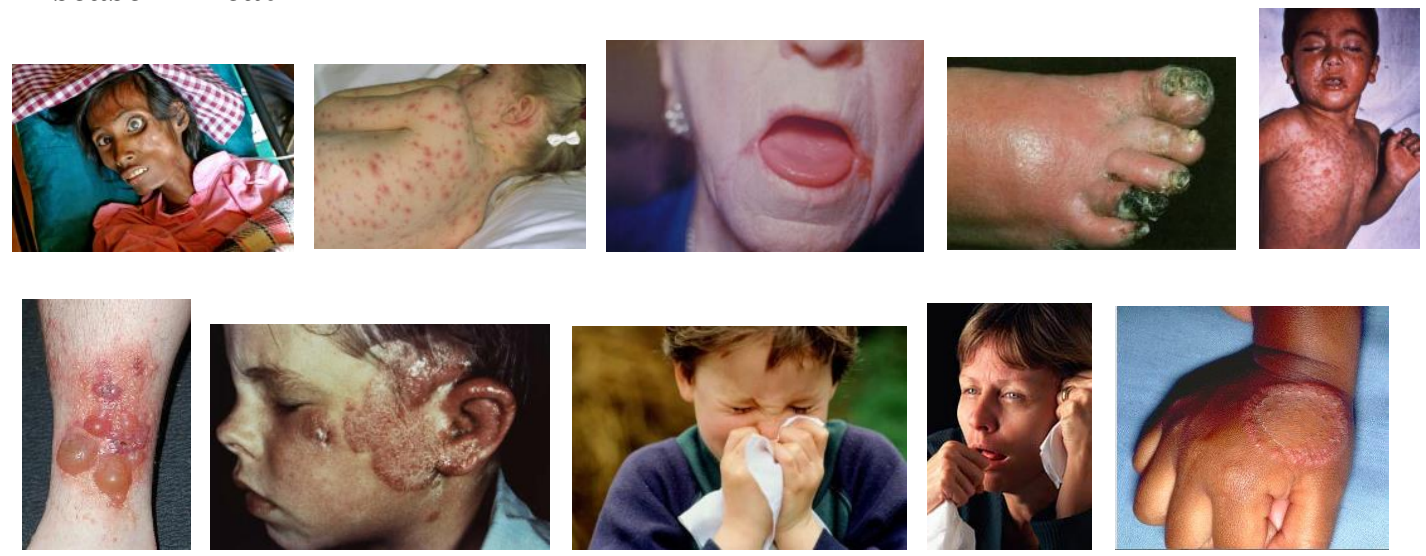

Non-Disease Threat
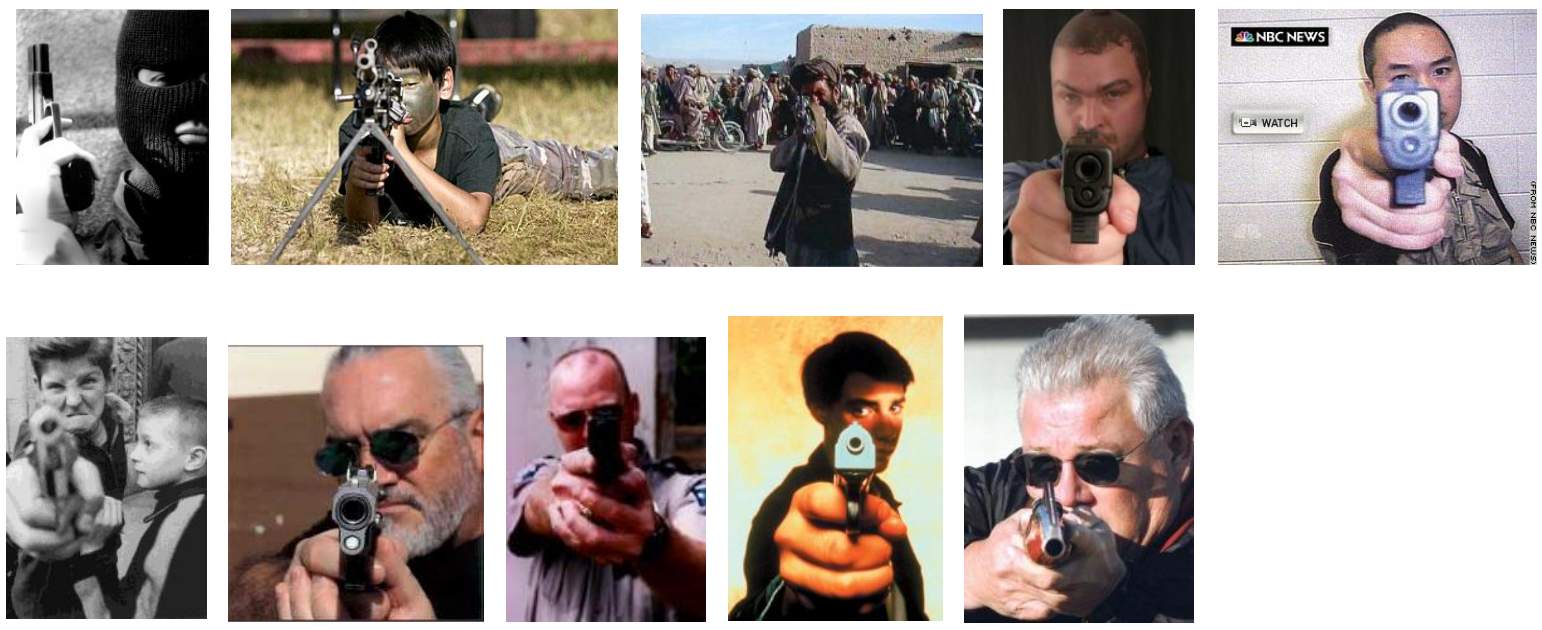

\section{Neutral}
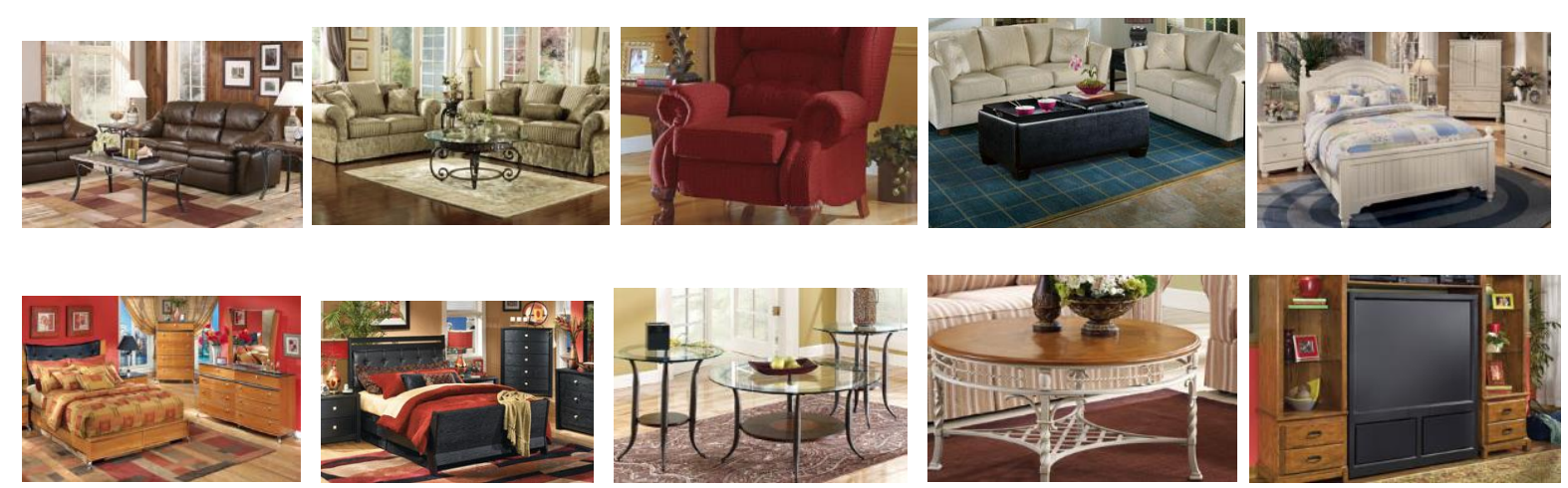